REVIEW ARTICLE

\title{
Next generation of heart regenerative therapies: progress and promise of cardiac tissue engineering
}

\author{
Miguel F. Tenreiro $\mathbb{D}^{1,2}$, Ana F. Louro $\mathbb{D}^{1,2}$, Paula M. Alves $\mathbb{D}^{1,2}$ and Margarida Serra $\mathbb{D}^{1,2 凶}$
}

The adult heart is a vital and highly specialized organ of the human body, with limited capability of self-repair and regeneration in case of injury or disease. Engineering biomimetic cardiac tissue to regenerate the heart has been an ambition in the field of tissue engineering, tracing back to the 1990s. Increased understanding of human stem cell biology and advances in process engineering have provided an unlimited source of cells, particularly cardiomyocytes, for the development of functional cardiac muscle, even though pluripotent stem cell-derived cardiomyocytes poorly resemble those of the adult heart. This review outlines key biologyinspired strategies reported to improve cardiomyocyte maturation features and current biofabrication approaches developed to engineer clinically relevant cardiac tissues. It also highlights the potential use of this technology in drug discovery science and disease modeling as well as the current efforts to translate it into effective therapies that improve heart function and promote regeneration.

npj Regenerative Medicine (2021)6:30; https://doi.org/10.1038/s41536-021-00140-4

\section{INTRODUCTION}

Cardiovascular diseases (CVDs) have been recognized as a major concern for global health among noncommunicable diseases ${ }^{1}$, and despite half-century of advances in cardiovascular science and preventive medicine, CVDs continue to yield high mortality and morbidity rates worldwide ${ }^{2}$. Revascularization interventions or pharmacological treatment allow in many cases to salvage the heart and prevent transplantation, even though such strategies are not recognized as a definitive cure ${ }^{3}$.

The adult human heart has a limited regenerative capacity ${ }^{4}$ and so regenerative medicine therapies have shed new hopes to repair or replace damaged hearts. The marginal benefits reported by cellbased $^{5-8}$ and cell-free ${ }^{9-11}$ approaches have grossly been associated with inappropriate delivery and retention, as well as ineffective therapeutic efficacy ${ }^{12}$. In the past decades, tremendous advances have been witnessed in the field of tissue engineering (TE), especially concerning stem cell engineering, the development of functional biomaterials and biomimetic scaffolds, and the implementation of biofabrication tools for the generation of complex biological structures with high resolution ${ }^{13}$. This progress has set a solid background to rethink current therapeutic approaches and design new bioengineering therapies ${ }^{14}$. Nonetheless, creating fully matured and functional cardiac tissue in vitro is still challenging. Despite this, it is becoming possible to robustly engineer miniaturized tissue versions able to compete, if not replace, commonly used platforms to assess drug safety and model human physiopathology to guide efficient drug discovery ${ }^{15}$.

This review highlights recent progress in cardiac stem cell bioengineering, with a particular focus on the identification of essential requirements for the design of clinically relevant human cardiac tissues: (i) the cell sources, (ii) bioinspired strategies to generate cardiomyocytes (CMs) with improved maturity, and (iii) biofabrication methodologies. The potential use of this technology in drug discovery science and disease modeling as well as the current efforts to translate it into effective heart regeneration therapies is also discussed.

\section{CELL SOURCES TO ENGINEER CARDIAC TISSUE}

Cardiac muscle is the functional unit of the heart, as it is involved in force generation and propagation of electrical signals through the CM-rich tissue to allow rhythmic pump contraction. Human cardiac muscle is composed of various cell types, the most abundant being CMs, pericytes, endothelial cells, and fibroblasts. The proportion of each cell population in the heart is still a matter of discussion, but there are evidences that CMs occupy the majority of the heart volume (roughly $75 \%$ ) and account for $30-40 \%$ of all cells ${ }^{16}$. State-of-the-art analyses of single-cell/nuclei RNA-sequencing transcriptomic data of six heart regions across several unrelated individuals have clarified that CMs are the most prevalent cardiac cell population in the heart, while also highlighting a surprising cellular heterogeneity among $\mathrm{CM}$, pericyte, endothelial, and fibroblast cell populations ${ }^{17}$. It appears that the cellular composition of cardiac muscle and its transcriptional signature varies across the heart in order to ensure that each anatomical region is highly specialized in a given function. Such complexity undeniably elevates the challenge of engineering artificial cardiac muscle.

Typically, engineering a contractile cardiac muscle requires foremost a source of functional CM. The first engineered cardiac tissue models relied on nonhuman cell cultures of different species, including cell lines of rat embryonic myoblasts $\left(\mathrm{H} 9 \mathrm{c} 2^{18}\right)$ and mouse $\mathrm{CMs}\left(\mathrm{HL}-1^{19}\right)$ as well as primary $\mathrm{CMs}$ isolated from chicken embryos ${ }^{20}$ and neonatal rats ${ }^{21}$. Despite limitations regarding human tissue availability, human adult CMs can be isolated from myocardial biopsies ${ }^{22}$; however, soon after isolation, CMs undergo a profound structural and functional remodeling, leading to cell dedifferentiation and loss of viability ${ }^{23}$. Recently, applying near-physiologic electromechanical stimuli during $24 \mathrm{~h}$ proved capable of inactivating CM remodeling after isolation ${ }^{24}$. Long-term culture (up to 4 months) of adult human myocardial muscle strips subjected to biomimetic stimuli similarly prevented pronounced CM remodeling, even though changes at the structural, mechanical, and gene expression levels were 
detected $^{25}$. Immortalized adult human ventricular CM cell lines have also been established by the fusion of primary cells with SV40-transformed human fibroblasts to create cardiac models ${ }^{26}$, but their poor recapitulation of human physiology discourages its use.

Due to their high self-renewal capacity and ability to generate any cell type of the human body, pluripotent stem cells (PSCs) are becoming an attractive alternative for cardiac TE. These PSCs can either be derived from the inner cell mass of the blastocyst, named embryonic stem cells (ESCs) ${ }^{27}$, or by forced expression of pluripotency genes through the delivery of the "reprogramming factors" Oct3/4, Sox2, Klf4, and c-Myc to somatic cells, termed induced PSCs (iPSCs) ${ }^{28,29}$. In particular, human iPSC (hiPSC) derivatives better capture patient-specific physiology ${ }^{30,31}$, besides surpassing the ethical concerns associated with human ESC (hESC). Acquired knowledge on stem cell biology together with the advances in PSC technology has allowed the establishment of robust differentiation protocols capable of obtaining every subtype of CM (ventricular, atrial, and pacemaker) with a high degree of purity ${ }^{32}$. Briefly, temporal modulation of the Wnt/ $\beta$-catenin signaling pathway is usually performed by first promoting activation for mesoderm formation and subsequent inhibition for cardiac specification ${ }^{33,34}$. The use of small molecules (e.g., CHIR99021, IWP2, IWR1) to control the Wnt/ $\beta$-catenin signaling pathway is currently preferred to growth factors/ recombinant proteins due to their better diffusion properties and moderate cost ${ }^{32,35,36}$.

A lot of effort has been put to evaluate the maturity state of human PSC-derived CMs (hPSC-CMs) and it is unanimous that, after differentiation, these cells are more similar to fetal rather than adult CMs (Fig. 1). Adult ventricular CMs display a remarkably organized structure with sophisticated intracellular organelles optimized for contraction. Among these components are densely packed aligned sarcomeres ${ }^{37,38}$ within a hypertrophic ${ }^{39}$ (usually) binucleated $^{40}$ cell; the sarcolemma and a vast network of transverse tubules (T-tubules) ${ }^{41,42}$ along the Z-lines to allow for fast depolarization; a functional longitudinal sarcoplasmic or endoplasmic reticulum (SR/ER) and terminal cisternae positioned near T-tubules adapted for quick calcium kinetics ${ }^{43}$; a high density of mitochondria near the contractile apparatus to improve ATP consumption ${ }^{44}$; and intercalated discs with well-developed cell junctions to promote electrical coupling ${ }^{45,46}$. hPSC-CMs have not shown this level of cellular complexity ${ }^{47-52}$, as they are less fit for contraction ${ }^{53,54}$ and are incapable of matching the metabolic demands of adult $\mathrm{CMs}^{55-57}$ needed for significant force generation $^{58-61}$, which is apparent in their negative force-frequency relationship (FFR) ${ }^{62,63}$ and minimal post-rest potentiation (PRP) ${ }^{62}$. Due to a dissimilar ion-channel density, hPSC-CMs exhibit a distinct action potential (AP) profile from adult $\mathrm{CMs}^{64,65}$, resulting in asynchronous and spontaneous beating rate ${ }^{66}$, less negative resting membrane potential (RMP) ${ }^{67,68}$, slower upstroke velocity ${ }^{69}$, and irregular AP duration ${ }^{67,69,70}$. In fact, the immature ultrastructure of hPSC-CMs not only affects calcium handling ${ }^{71}$ due to an under-developed excitation-contraction coupling (ECC) machinery ${ }^{50,51,62,72}$ but it also slows AP propagation ${ }^{73,74}$ owing to the lack of organized electromechanical junctions compared to adult cardiac tissue $e^{75,76}$. Furthermore, these differences are seen at a transcriptomic level, with hPSC-CMs showing preferential expression of fetal sarcomeric gene isoforms (myosin heavy chain: $M Y H 6$ $>$ MYH7; troponin I: TNNI1 > TNNI3; titin: TTN-N2BA > TTN-N2B) as well as lower expression of genes related with electrophysiological function (e.g., KCNJ2, KCND3, KCNJ8, KCNH7, SCN5A, HCN4, GJA1, JPH2) and ECC (e.g., CACNA1C, RYR2, ATP2A2, CASQ2, SLC8A1, PLN, ITPR3, CAV3, BIN1) ${ }^{77}$.

The maturation defects of hPSC-CMs have gained increased attention over the past decade since their immaturity greatly limits their application in several areas of precision medicine. A better understanding of cardiac development has allowed the

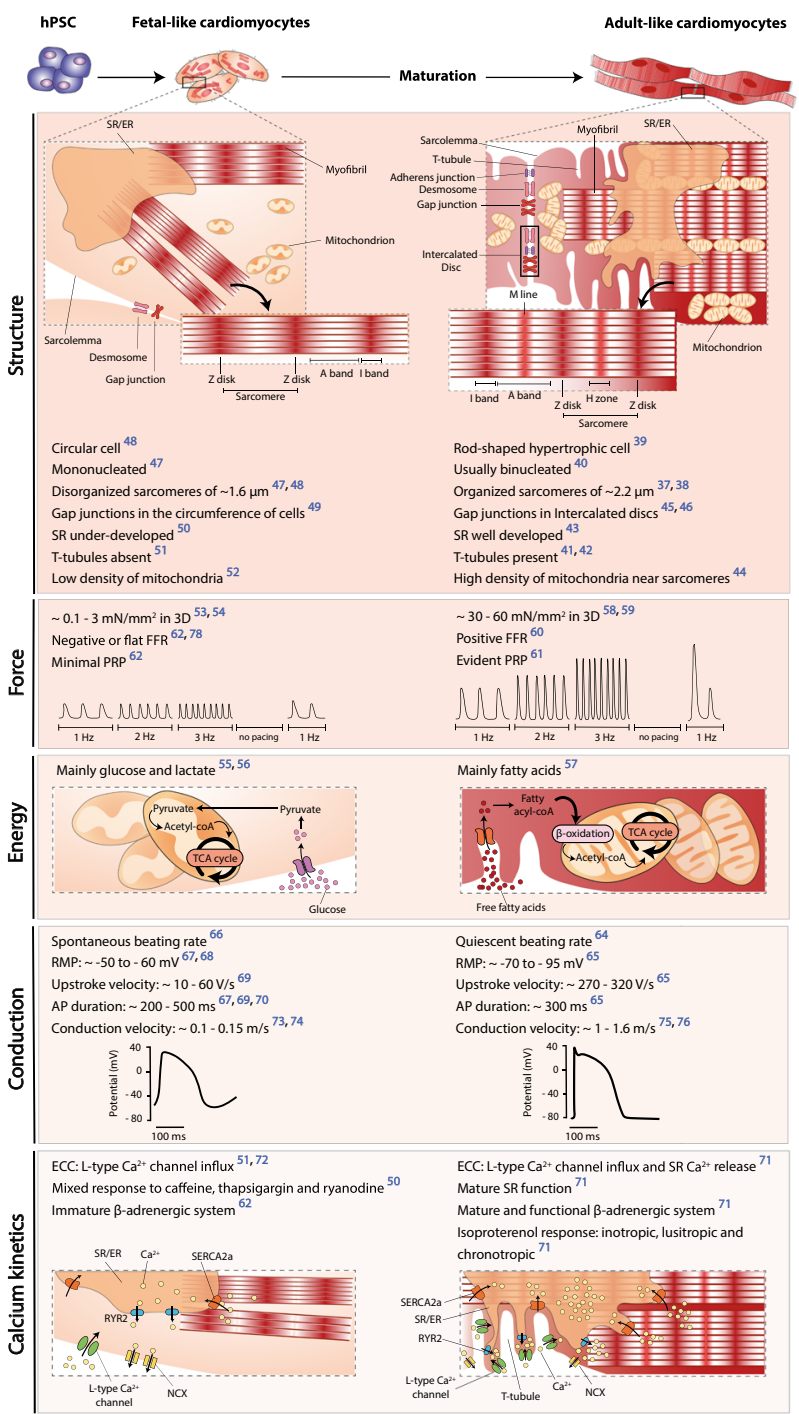

Fig. 1 Differences between human pluripotent stem cell-derived cardiomyocytes (hPSC-CMs) and ventricular cardiomyocytes from adult cardiac tissue. hPSC-CMs obtained from the differentiation of pluripotent stem cells present fetal-like features in respect to structural and ultrastructural organization, contractile force, metabolism, and electrophysiological function. AP action potential, ECC excitation-contraction coupling, FFR force-frequency relationship, hPSCs human pluripotent stem cells, NCX sodium-calcium exchanger, PRP post-rest potentiation, RMP resting membrane potential, RYR2 ryanodine receptor type 2, SERCA2a sarcoplasmic/endoplasmic reticulum calcium-ATPase $2 \mathrm{a}$, SR/ER sarcoplasmic/endoplasmic reticulum.

identification of relevant players that partake in the transformation of the fetal heart into a competent and efficient organ. Complete maturation of hPSC-CMs has yet to be reported; however, nature-inspired biological strategies and/or engineering solutions proposed over the past few years have undoubtedly made significant contributions to improve the maturation of these cells.

\section{BIOINSPIRED STRATEGIES TO IMPROVE HPSC-CM MATURITY}

During cardiac development, several environmental factors including mechanical forces, electrical stimuli, biochemical factors' gradients, extracellular matrix (ECM) remodeling, and heterotypic interactions drive CM maturation ${ }^{78}$. Maturation is the last phase of 


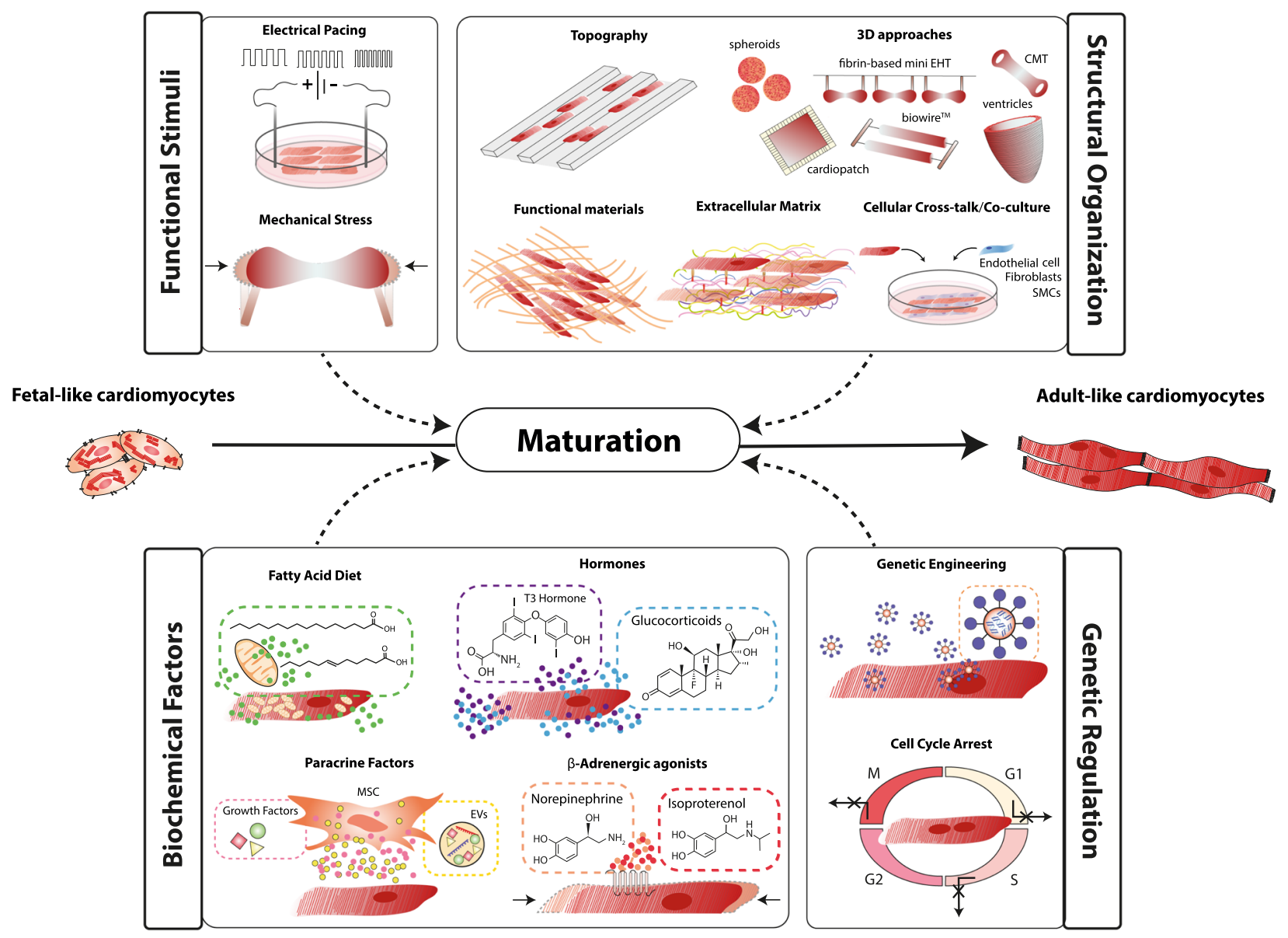

Fig. 2 Bioengineering strategies to improve the maturation of cardiomyocytes derived from human pluripotent stem cells (hPSC-CMs). hPSC-CMs can develop adult-like features with training based on functional stimuli, cues to guide their structural organization, relevant biochemical factors, and by manipulating the genetic program. CMT cardiac microtissue, EHT engineered heart tissue, EV extracellular vesicle, MSC mesenchymal stem cell, SMC smooth muscle cell, T3 triiodothyronine.

cardiac development and corresponds to a series of orchestrated phenotypic events that prepare the fetal heart for efficient and competent life-long pumping function. Some of these events are initiated in utero, such as physiologic hypertrophic growth, sarcomeric isoform switch, cell cycle exit, and a glycolytic-tooxidative metabolic switch ${ }^{79}$. However, the highly specialized cellular machinery of adult CMs only fully develops years after birth $^{46}$. Even though prolonging time in culture of hPSC-CMs (up to 1 year) improves structural and functional maturation ${ }^{47,80,81}$, these cells still show signs of a fetal phenotype. Early-stage hPSCCMs transplanted in animal hearts were able to acquire adult-like features ${ }^{82,83}$, providing evidence of their plasticity and ability to be matured if the right environment is ensured. By attempting to mimic nature, various bioengineering approaches have been proposed to promote hPSC-CM maturation (Fig. 2).

\section{Functional stimuli}

In cardiac tissue, CMs are responsive to external electrical stimuli and mechanical forces to perform their function, and these are initiated early during cardiac embryogenesis.

Electrical excitability is a key feature of CMs, dictating electromechanical coupling. The effect of exogenous electrical stimuli on CM maturation was first tested using neonatal rat CMs seeded in collagen sponges and exposed to pulse waves at $1 \mathrm{~Hz}$ for up to 8 days, resulting in cell alignment and elongation along the electrical field as well as the formation of intercalated discs with gap junctions expressing connexin- $43^{84,85}$. Both neonatal rat
$\mathrm{CMs}^{86,87}$ and hPSC-CMs ${ }^{88}$ have also shown a more mature phenotype after being stimulated with pulse frequencies $>1 \mathrm{~Hz}$. Interestingly, applying a supraphysiological pacing regimen up to 1 week (increasing pulse frequency from 1 to $6 \mathrm{~Hz}$ ) to hPSC-CMs seeded in a collagen matrix markedly contributed to cell alignment, establishment of an organized contractile ultrastructure, improved SR/ER function, and electrophysiological profile ${ }^{89}$.

In turn, mechanical stress induces CM rhythmic contraction, which has a direct impact on physiologic hypertrophic growth, cell elongation, tissue alignment, and force generation. By casting neonatal rat $\mathrm{CMs}$ in a ring-shaped mixture of collagen and Matrigel ${ }^{\circledast}$ exposed to mechanical stretch, it was demonstrated for the first time the importance of applying stress to induce both ultrastructural and functional maturation of $\mathrm{CM}^{90}$. Since cylindrical casting molds provided little tissue deflection, mechanical stimulation via cyclic stretch is now achieved using fibrin-based hydrogels in flexible posts ${ }^{91}$ or in thin elastomeric frames ${ }^{92}$, allowing for auxotonic muscle contraction. These technologies were first established using rodent cells, but were successfully translated to hPSC-CMs ${ }^{62,93}$. hPSC-CMs show alignment along the hydrogel and sarcomeric organization ${ }^{62}$, a metabolic shift towards fatty acid consumption ${ }^{94}$, improved calcium drug sensitivity ${ }^{95}$, and force development ${ }^{62}$, even though the force generated (up to $\sim 1$ $\mathrm{mN} / \mathrm{mm}^{2}$ ) is still relatively inferior to those of adult cardiac muscle strips $\left(\sim 30-60 \mathrm{mN} / \mathrm{mm}^{2}\right)^{58,59}$. More recently, modulation of cyclic $^{96}$ and passive stretching ${ }^{97}$ of hPSC-CMs revealed an impact on tissue functional performance, indicating that there might be ways to further improve tissue force generation. Of note, more 
complex systems simulating the hemodynamic loads of the cardiac cycle have also been used to improve hPSC-CM maturation ${ }^{98}$.

In addition, CMs can be electrically and mechanically stimulated simultaneously ${ }^{99-101}$, thereby enhancing maturation. In a recent work, hPSC-CMs subjected to combined cyclic stretch in elastomeric posts and a supraphysiological pacing regimen of 2 weeks (increasing pulse frequency from 2 to $6 \mathrm{~Hz}$ ) resulted in these cells displaying adult-like features never reported before, such as a vast network of T-tubules, inotropic response to isoproterenol, a positive FFR, and noticeable PRP ${ }^{101}$. This represents the most advanced strategy reported for hPSC-CM maturation so far, although these more mature hPSC-CMs still presented inadequate contractile force generation ability (up to $\sim 4 \mathrm{mN} / \mathrm{mm}^{2}$ ) when compared to adult counterparts.

\section{Structural organization}

Cardiac tissue function depends on a highly anisotropic architecture with orderly aligned myofibrils along the direction of muscle contraction, which is impossible to replicate when culturing CMs on standard and simple plastic two-dimensional (2D) surfaces. Promoting this level of cellular organization can be achieved using grooved patterned substrates where hPSC-CMs already showed elongation along the line patterns, electromechanical coupling with neighboring $\mathrm{CMs}$, and electrophysiological improvements ${ }^{68,102}$. Of note, substrate stiffness, groove width, and cell density seem to have an impact on the structural maturation of hPSC-CMs ${ }^{103}$. In fact, the stiffness of a substrate can by itself modulate the mechanical load experienced by hPSC-CMs, affecting the cytoskeleton organization ${ }^{104}$ and contractility ${ }^{102}$. In addition, various natural (collagen ${ }^{20,89}$, fibrin ${ }^{91}$, and Matrigel ${ }^{90,105}$ ) or synthetic (polyesters ${ }^{106,107}$, polyethylene glycol $^{108}$, polylactones $^{109}$, and elastomers ${ }^{110}$ ) biomaterials can be used to guide cardiac tissue structural organization, even though their properties (mechanical, topographical, and biocompatibility) influence the degree of maturation obtained. Unfortunately, none of these biomaterials can fully recapitulate the architecture and functional composition of the cardiac ECM. Still, artificial ECM ${ }^{111}$ or decellularized myocardial ECM $\mathrm{E}^{12-114}$ has been reported to promote hPSC-CM maturation without biomimetic regimens.

Throughout cardiac development, CMs are in direct contact with other cell types. Even though non-myocytes occupy a relatively small volume in cardiac tissue $(\sim 25 \%)$, they are necessary for CM survival, contractile performance, cell-cell communication, vascular supply, and ECM deposition ${ }^{115}$. Coculture of hPSC-CMs with endothelial cells, either on Matrigel ${ }^{\oplus}$-coated surfaces ${ }^{116,117}$ or Matrigel hydrogels $^{118}$, resulted in higher expression of proteins related with CM maturity ${ }^{116}$, contributed for ultrastructural organization $^{116,117}$, improved contractility ${ }^{118}$, and calcium handling ${ }^{116,118}$.

Assembling hPSC-CMs into three-dimensional (3D) tissues greatly improves cell-cell communication with more physiological relevance, promoting structural, metabolic, and functional maturation. Engineered tissues can assume various architectures, such as cell sheets ${ }^{119}$, spheroids ${ }^{120,121}$ and organoids ${ }^{122}$, muscle strips in the form of rings ${ }^{90,123}$, ribbons ${ }^{63,124}$ or cables $^{89,92}$, patches ${ }^{125,126}$, and even ventricle analogs ${ }^{127}$. A recent study using a patch platform reported, without exogenous stimulation, advanced hPSC-CM mechanical maturity with a contractile force closer to adult ventricular tissue $\left(\sim 17-22 \mathrm{mN} / \mathrm{mm}^{2}\right)^{126}$, even though it had slightly negative FFR and an organized ECC apparatus without pronounced T-tubulation. Nonetheless, 3D approaches are extremely versatile and can be combined with other strategies to enhance maturation and tissue performance (e.g., intensive electrical training ${ }^{101}$, dynamic culture ${ }^{92}$, coculture with other cell types ${ }^{128-130}$ ). For instance, culturing fibroblasts and hPSC-CMs in muscle strips with a ratio of 1:3 improved cell-cell communication and tissue remodeling ${ }^{128}$, in addition to also contributing to hPSC$\mathrm{CM}$ maturation. Intriguingly, the electrical pacing of hPSC-CM muscle strips in flexible posts requires a supporting network of fibroblasts, otherwise the tissue loses its integrity when electrically stimulated $^{101,131}$. Due to the increasing relevance of 3D maturation-based strategies to the field, we will further detail $3 \mathrm{D}$ tissue fabrication methodologies in this review (see "Fabrication of biomimetic cardiac tissues").

\section{Biochemical factors}

Several soluble and biological factors have been proposed to induce hPSC-CM maturation, such as the type of carbon substrate and hormones. In vivo, CM maturation is characterized by a metabolic shift from glycolysis to preferential fatty acid oxidation, a consequence of cellular hypertrophy and higher energetic demands ${ }^{56}$. hPSC-CMs display metabolic substrate plasticity that can be modulated to promote fatty acid $\beta$-oxidation ${ }^{132,133}$. Our group has demonstrated that hPSC-CM structural, functional, and metabolic maturation can be improved in fatty acid-containing medium with galactose to prevent lipotoxicity ${ }^{134}$, when compared with glucose-rich medium. Among hormones, triiodothyronine (T3) is essential during cardiac development ${ }^{135}$, and has revealed to play a key role in inducing hPSC-CM maturation ${ }^{136}$. Furthermore, by combining T3 with dexamethasone, a glucocorticoid hormone, noticeable improvements of SR/ER contribution in ECC and T-tubulation were detected, which otherwise was not seen with each hormone alone ${ }^{137}$.

Biological factors such as those secreted by neighboring cells within the cardiac niche can also positively impact CM maturation state. Recently, hPSC-CM and mesenchymal stem cell (MSC) coculture promoted cellular cross-talk via the release of cytokines and extracellular vesicles capable of modulating hPSC-CM sarcomeric organization, electrophysiology, and metabolism ${ }^{138}$. Another study used a spherical microtissue containing hPSC-CMs as well as cardiac-specific endothelial cells and fibroblasts, and revealed that this tri-cellular interaction upregulated the CAMP/ $\beta$-adrenergic pathway ${ }^{130}$. An increase in intracellular CAMP levels consequently affected the assembly of connexin-43 gap junctions, besides improving hPSC-CM structural, contractile, metabolic, and electrophysiologic features. Taken together, by identifying key biological factors capable of inducing CM maturation, these studies underline the importance of heterotypic cell interaction in the cardiac microenvironment.

A less conventional approach to induce CM maturation has been the use of $\beta$-adrenergic agonists, namely norepinephrine ${ }^{139}$, isoproterenol $^{140}$, and phenylephrine ${ }^{141,142}$. These compounds are capable of causing structural changes and cellular hypertrophy by inducing intensive workload, even though these effects are likely dose-dependent to avoid pathological remodeling and cytotoxicity.

\section{Genetic regulation}

Adult CMs have defined gene regulation mechanisms, ultimately dictating cardiac tissue phenotype and maturation. Overexpressing KCNJ2 during differentiation resulted in hPSC-CMs with hyperpolarized RMP and eliminated the AP proarrhythmic trait during repolarization, even though calcium handling and structural features were not improved due to early loss of automaticity ${ }^{143}$. In turn, calcium kinetics were significantly improved in hPSC-CMs by forced expression of CSQ2 $2^{144}$. Instead of manipulating the transcriptome, altering the epigenetic state of hPSC-CMs using histone $\mathrm{H} 3$ deacetylase inhibitors also showed to promote adult-like expression of calcium handling and conduction genes $^{145,146}$, even though reports regarding electrophysiologic improvements are inconsistent. Priming cells with polyinosinicpolycytidylic acid at the cardiac progenitor stage of differentiation strikingly accelerated structural, electrical, and metabolic maturation of hPSC-CMs, and this acceleration of the developmental 


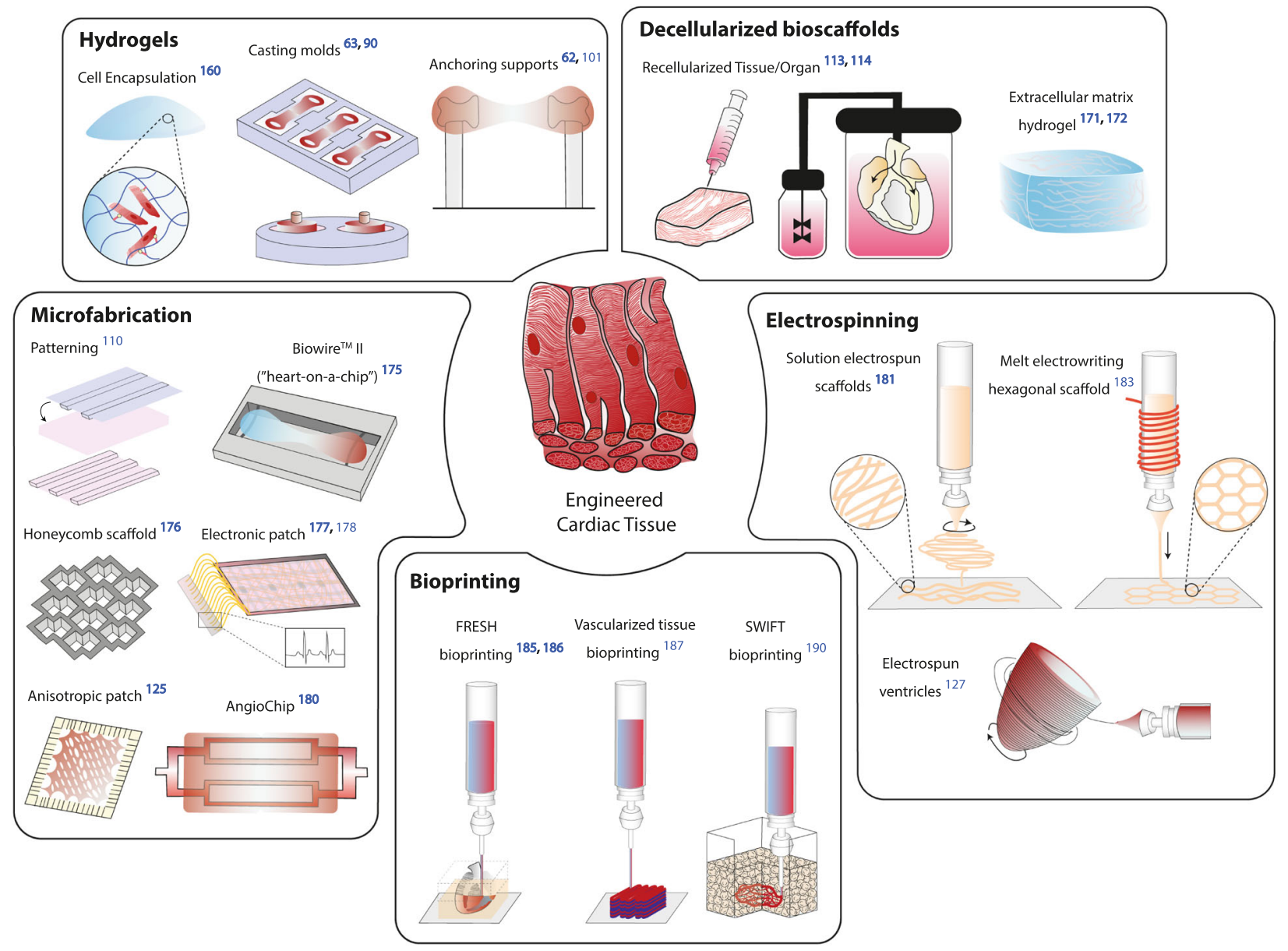

Fig. 3 Overview of state-of-the-art biomimetic cardiac tissues and corresponding fabrication approaches. Engineered cardiac tissues range from hydrogels capable of encouraging muscle fiber organization, decellularized scaffolds for tissue/organ bioengineering, microfabricated systems for promoting tissue anisotropy and vascularization, electrospun fibers for close mimicry of tissue architecture, and even printable tissue/miniaturized organs. FRESH Freeform Reversible Embedding of Suspended Hydrogels, SWIFT Sacrificial Writing Into Functional Tissue.

clock was associated with increased H3K9ac activation that promoted earlier transcription of cardiac myofilament genes and the Notch ligand $J A G 1^{147}$. In another study, the inhibition of aberrantly upregulated factors involved in aerobic glycolysis promoted an hPSC-CM metabolic shift towards oxidative phosphorylation, and consequently features of functional maturation $^{133}$. Moreover, the comprehensive analyses of micro-RNA (miR) levels throughout hPSC-CMs' prolonged culture have identified those that become differentially expressed ${ }^{148,149}$, suggesting alternative routes to modulate gene expression to induce maturation. Let-7 family miRs ${ }^{150}$, miR-1 ${ }^{151,152}$, miR-499 ${ }^{152}$, and mir-200c ${ }^{153}$, and a combination of miRs (miR-125b, miR-199a, miR221 , and miR-222 $)^{154}$ have proved useful to improve hPSC-CM maturation features.

Soon after birth, CMs become nonproliferative cells due to extensive hypertrophic growth and remarkable sarcomere reorganization hindering cytokinesis. Ultimately, these changes result in an increase in binucleation and polyploidy. A recent work revealed that knockout of the ECM protein agrin in neonatal mice resulted in the loss of $\mathrm{CM}$ proliferation alongside sarcomere assembly and maturation ${ }^{155}$. As expected, administration of agrin to hPSC-CMs avoided loss of proliferation, but negatively affected gene expression and conduction velocity ${ }^{155}$. By promoting cell cycle cessation, mitomycin C treatment similarly increased sarcomerogenesis in neonatal rat-derived PSC-CMs ${ }^{156}$. Further work will be needed to elucidate how cell cycle arrest can promote hPSC-CM maturation other than structural improvements.

\section{FABRICATION OF BIOMIMETIC CARDIAC TISSUES}

Inducing the maturation of hPSC-CMs is still a challenge in the field of cardiac TE; however, considerable progress has been achieved by seeking innovative approaches. Moving from simple beating cells to physiologically relevant tissues will require further efforts to engineer the complexity of the in vivo conditions. In the following section, we highlight the most promising techniques adopted to date to grow biomimetic cardiac tissue in the laboratory (Fig. 3). A more detailed overview of these current biofabrication methodologies, their advantages and disadvantages for translation to TE and regenerative medicine fields can be found in ref. ${ }^{157}$.

\section{Hydrogel method}

Hydrogels are the most widely used polymers for cardiac TE due to their biocompatible physical and chemical properties, including high water content, efficient gas and mass transfer (i.e., adequate exchange of oxygen and metabolites), ability to be molded into several geometries, easy to be modified/functionalized in order to include cell-binding sites (e.g., RGD integrin-binding domains and ECM proteins), and scalability ${ }^{158,159}$. Cardiac tissues can be 
fabricated by joint gelation of cells and a polymer ${ }^{160}$ or by having a casting mold to confine the cells in a given polymeric organization $^{63,90}$. Typically, natural hydrogels $s^{89,91,101}$ are preferred for engineering cardiac tissues by promoting cell assembly and subsequent maturation, but functionalized synthetic polymers ${ }^{106}$ or hybrid biomaterials ${ }^{161,162}$ are also suitable options. Cells often self-organized within hydrogels in ways that are different from those found in native tissues, and so it can be advantageous to tailor the hydrogel's microarchitecture to encourage anisotropic rather than isotropic tissue growth, which can be done by relying on simple methods such as freeze-drying, solvent casting, gas foaming, and unidirectional freezing ${ }^{163}$. Hydrogels are generally soft, with inferior mechanical compliance in comparison to the native heart tissue. Anchoring supports are useful not only to retain hydrogel conformation but also to provide a more adequate mechanical load to $\mathrm{CM}^{62,101}$ and facilitate force measurement ${ }^{87,131,164}$. Impressively, the resulting tissues can be several millimeters long $\left(\sim 7-9 \mathrm{~mm}^{62,101}\right)$.

\section{Decellularized bioscaffolds}

The main aim of decellularization methodologies is to generate scaffolds with native ECM ultrastructure and composition while removing all cells and genetic material present in the native tissue. Several decellularization protocols have been described for nearly all tissues and organs in the body, and include physical, chemical, and/or enzymatic approaches. Discussing the effects of these agents is beyond the scope of this review (reviewed in refs. ${ }^{165,166}$ ); however, it is important to highlight that the choice of optimal protocol for tissue decellularization depends on many factors, including tissue source, cellularity, composition, density, thickness, and donor age, and these overall impact the efficiency of the decellularization process and the resultant ECM composition.

Cardiac tissue or whole heart decellularization is typically accomplished by using ionic and nonionic detergents. Decellularization of a whole rat heart through perfusion ${ }^{167}$ and of thin slices of human myocardial tissue ${ }^{168}$ using sodium dodecyl sulfate (SDS) resulted in lower DNA content and maintenance of native ECM structure, when compared with approaches using other chemicals. Typically, decellularizing human cardiac tissue ${ }^{114}$ requires a higher SDS exposure time than animal-derived cardiac tissue ${ }^{169,170}$, and the reasons for this can be manyfold, such as higher collagen and lipidic content, a stiffer ECM, or donor age. Because of this, it is becoming clear that interspecies differences may hinder the use of decellularization protocols optimized in animals for human tissue. The most immediate application of decellularized myocardial sheets $\left(\sim 200-400 \mu \mathrm{m}\right.$ thickness $\left.{ }^{113,114}\right)$, muscle strips $(15 \mathrm{~mm}$ length, $2.5 \mathrm{~mm}$ diameter ${ }^{114}$ ), or whole heart ${ }^{114}$ is scaffold recellularization. Even though myocardial sheets and strips were successfully recellularized with CMs, recellularization of a human heart is an unmet goal and the best attempt so far has been an $\sim 500$ million CM intramyocardial injection to an $\sim 5 \mathrm{~cm}^{3}$ volume of an acellular heart scaffold ${ }^{114}$, which is way below the billions of CMs necessary to repopulate this organ. Nevertheless, isolated ECM can be broken down, solubilized with pepsin, and then assembled into a nanofibrous hydrogel ${ }^{171}$, inevitably destroying the structural and mechanical architecture of the ECM scaffold. Interestingly, decellularized human cardiac ECM requires additional steps of lipid removal before hydrogel formation ${ }^{172}$. Soluble ECM in 3D settings promoted CM maturation ${ }^{112}$, but has a wider application for scaffold functionalization and has shown versatility to be implemented in advanced manufacturing techniques $^{169,173,174}$. Despite its promising applications, decellularized human cardiac ECM is scarce due to donor organ shortage, and therefore research often relies on off-the-shelf animal-derived matrices.

\section{Microfabrication}

Advances in microfabrication have allowed detailed engineering of material features in order to resemble tissue in vivo conditions. In particular, photolithography enables the precise transfer of geometrical shapes from a mask onto a substrate to obtain defined topographies for anisotropic tissue organization, while with soft lithography, substrates can be designed to include microchannels and complex microfluidic networks that are useful to recreate tissue vasculature. However, manufacturing such platforms can be time consuming, requires specialized equipment and facilities, and production may be costly.

The simpler systems use microfabrication to pattern material substrates, so as to ease CM spreading and sarcomere axial alignment. For instance, one of the initial attempts used polydimethylsiloxane (PDMS) thin films that contained an array of alternating $20-\mu \mathrm{m}$-wide lines, and these grooves substantially improved CM alignment and showed compliance during the cardiac cycle ${ }^{110}$. Currently, more sophisticated patterned culture platforms have been established, which synergize well with biochemical cues. In fact, circular chambers (ranging from 200 to $600 \mu \mathrm{m}$ diameter), obtained through plasma etching of polyethylene glycol substrates, allowed geometrical confinement of hiPSC colonies and mediated morphogenesis of cardiac microchambers during differentiation ${ }^{122}$. Patterned culture chambers can also be useful to support hydrogel-based cardiac tissue formation ( 3-4 mm length) and its long-term maturation (e.g., Biowire ${ }^{\mathrm{TM}}$ ${ }^{1{ }^{175}}$ ). Nonetheless, microfabrication methodologies can be harnessed to create complex tissues similar to the native myocardium. Pioneering work used laser microablation of poly(glycerol sebacate) membranes to produce an accordion-like honeycomb scaffold, which was designed by overlapping two $200 \mu \mathrm{m} \times$ $200 \mu \mathrm{m}$ squares at a $45^{\circ}$ angle ${ }^{176}$. The unconventional design was purposely conceived to closely resemble the heart anisotropy, and proved successful in guiding CM alignment and improving their mechano-microenvironment. In another study, a porous photomask $(2.5 \mathrm{~cm} \times 2.5 \mathrm{~cm})$ was crafted based on cardiac fiber orientation of a specific epicardial plane retrieved from a $3 D$ reconstruction of the human ventricle ${ }^{125}$. This mask was used to mold a PDMS substrate that allowed a patch-like cardiac tissue formation in a developmentally mimetic fashion. More contemporary improvements have advanced even further cardiac patch design by using conductive biomaterials with built-in nanoelectronics ${ }^{177,178}(\sim 20 \mathrm{~mm} \times 5 \mathrm{~mm})$, which are practical for online electrophysiological recording and electromechanical pacing.

Simple fabrication technologies, such as hydrogels, are unable to integrate organized vasculature within engineered tissue; however, microfabricated cardiac tissues can be assembled with perfusable and highly branched endothelialized channels ${ }^{179,180}$ by relying on simple soft lithography approaches. In particular, the AngioChip device $(5 \mathrm{~mm} \times 3.1 \mathrm{~mm} \times 150-300 \mu \mathrm{m})$, a biodegradable elastomer assembled with layer-by-layer 3D stamping, reported unprecedented functional vascularization of cardiac tissues, allowing perfusion through an open lumen, capillary outgrowth, enhanced permeability, structural integrity, and matrix remodeling ${ }^{180}$.

Despite the lack of standardized culture modes across these systems, remarkable tissue-level complexity can be achieved, as well as adequate tissue function for high-throughput analysis ${ }^{175}$.

\section{Electrospinning}

Electrospinning is a versatile method to fabricate ECM-like scaffolds as braided, woven, or knitted fibrous networks, from both naturally derived and synthetic materials. In solution electrospinning, the polymer is gravitationally forced to pass through an electrostatic field to generate a fibrous meshwork. Even though electrospun fibers frequently display inappropriate porosity compromising cell spreading and infiltration, certain 
parameters, like voltage and polymer flow rate, can be readily adjusted to fabricate the desired fibrous architecture. We have used solution electrospinning to produce poly( $\varepsilon$-caprolactone) (PCL) electrospun scaffolds $(\sim 2 \mathrm{~cm} \times 2.5 \mathrm{~cm} \times 229 \mathrm{~nm})$, and the resulting fibers displayed an appropriate architecture that allowed $\mathrm{CM}$ alignment ${ }^{181}$. Furthermore, coating the scaffold with piezoelectric microfibers provided suitable electrical stimuli to the cells.

Over the past few years, there have been proposed other electrospinning-based techniques, which aimed at improving certain drawbacks of the traditional method, such as dependency on the polymer's solution conductivity, slow deposition rates, and imprecise fabrication of 3D structures. Producing nano- to submicrometer scale fibers with a fast rate can be achieved using a high-speed rotating reservoir that propels centrifugal polymer deposition in a cylindrical collector ${ }^{182}$. The resulting polyester scaffold ( $25 \mathrm{~mm}$ diameter) was shown to promote CM alignment. A more recent approach used an ellipsoidal mandrel to collect electrospun $\mathrm{PCL}$ gelatin-coated nanofibers ${ }^{127}$. The ventricleinspired structure (truncated ellipsoid; $4.5 \mathrm{~mm}$ diameter, $9 \mathrm{~mm}$ height) promoted CM laminar alignment, even though the ejection fractions and contractile work were $\sim 50-250$ and $\sim 10^{8}$ smaller than the physiologic values for a human ventricle, respectively. Another promising methodology is melt electrospinning (also known as melt electrowriting), which allows a more controlled scaffold fabrication by precise collector or nozzle positioning, similar to 3D printing. Despite being unsuitable for cell encapsulation purposes, a relatively recent study demonstrated that a foldable PCL melt electrospun hexagonal-shaped scaffold $(8 \mathrm{~mm}$ diameter, $300 \mu \mathrm{m}$ thick) contributed to CM alignment and maturation ${ }^{183}$. Future improvements to these techniques or integrating existing expertise in multistep biofabrication approaches could allow their widespread use for cardiac TE.

\section{Bioprinting}

Bioprinting is an emerging biofabrication method based on precise layer-by-layer deposition of biomaterials, biochemicals, and living cells, collectively named bioinks ${ }^{184}$. High-fidelity tissue and organ surrogates can be generated with the aid of medical imaging modalities, namely computed tomography and magnetic resonance imaging.

Synthetic materials were amongst the first to be printed, but now research focuses on using naturally derived materials, such as ECM hydrogels derived from decellularized tissue. In particular, cardiac-specific ECM-derived bioinks, when pressure extruded through a printing nozzle, have successfully generated grid-type structures in the form of rectangles ${ }^{169}(\sim 5 \mathrm{~mm} \times 5 \mathrm{~mm} \times 1 \mathrm{~mm})$ or circles $^{173}$ (10 mm diameter, $0.6 \mathrm{~mm}$ thick), such that it resembles a patch. Extrusion bioprinting is one of the most popular methods to directly print cell-laden hydrogel-based cardiac tissue in an organized and reproducible fashion, besides allowing printing with high cell densities ${ }^{184}$, which is a necessary requirement to print more complex structures. Aside from the above-mentioned simple tissue constructs, extrusion bioprinting can be used to create more complex tissue models, but freeform printing of such structures requires a supporting sacrificial material. A novel method termed "FRESH" (i.e., Freeform Reversible Embedding of Suspended Hydrogels) achieved extrusion-based freeform printing by embedding the bioink in a thermally reversible supportive hydrogel during printing ${ }^{185,186}$, and this allowed fabrication of high-resolution 3D anisotropic structures, including a beating ventricle (truncated ellipsoid; $5.7 \mathrm{~mm}$ diameter, $8 \mathrm{~mm}$ height) and an acellular neonatal-sized heart analog $(37 \mathrm{~mm}$ diameter, $55 \mathrm{~mm}$ height $)^{186}$. Another study used a similar approach, but with an enzymatic or chemical reversible supportive hydrogel, and was able to print a heart-shaped model $(14 \mathrm{~mm}$ diameter, $20 \mathrm{~mm}$ height) using an omental ECM biomaterial in combination with
CMs and endothelial cells ${ }^{174}$. Further improvements are needed to create a functional tissue since the cells were reported to be arranged as nonaligned aggregates after 1 day in culture.

Another advantage of bioprinting is the possibility of creating tissues with inherent perfusable vasculature ${ }^{187-189}$. Direct extrusion-based bioprinting was used to manufacture one of the first vascularized cardiac tissues, in which the resulting endothelialized grid-type structure $(\sim 5.5 \mathrm{~mm} \times 3.5 \mathrm{~mm} \times 0.75 \mathrm{~mm})$ was seeded with CMs and perfused with a microfluidic device ${ }^{187}$. Bioprinting a vascular network greatly improves the thickness these tissues can achieve without becoming necrotic $\left(>1 \mathrm{~cm}^{188}\right)$, but they often lack physiologically relevant cell numbers $\left(\sim 1 \times 10^{8}\right.$ cell $/ \mathrm{mL}$ ). A new innovative method termed "SWIFT" (i.e., Sacrificial Writing Into Functional Tissue) relies on assembling up to 400,000 spheroids/organoids as a tissue matrix and then extrudes a sacrificial ink throughout the compact granular tissue, which can later be evacuated and the resulting lumen perfused ${ }^{190}$, despite lacking robustly endothelialized channels. This clever technique allowed the assembly of a cardiac tissue with 240,000 million cells $(6 \mathrm{~mm}$ top width, $4.2 \mathrm{~mm}$ bottom width, $12 \mathrm{~mm}$ height, $4.2 \mathrm{~mm}$ depth) that fuse and beat synchronously over 7 days in culture. Stereolithography, a higher resolution bioprinting methodology that uses a light projector to solidify layer-by-layer the bioink, has recently been used to create entangled vascular networks to perfuse an alveolar sac model ${ }^{189}$. Due to its exceptional level of functionality, translating this approach to cardiac TE could also be worthwhile.

It is important to take into account that additive printing with a high level of detail requires a slow printing velocity, affecting the scalability of current methods. To overcome this limitation, a recent study demonstrated that tissue can be printed within seconds with high fidelity by irradiating multiple light patterns representing projections of the object to be printed onto a photopolymer ${ }^{191}$. Despite being a promising technology, its potential use for cardiac TE still needs to be evaluated.

\section{UNLOCKING CARDIAC TISSUE ENGINEERING FOR PRECISION MEDICINE}

The advances in hPSC biology together with the development of novel materials and biofabrication methods have enabled the production of patient-specific cardiac tissues, which in turn opens up the possibility of fulfilling the promise of personalized medicine (Fig. 4). This section includes a brief overview of the current state-of-the-art applications of cardiac TE for drug discovery, disease modeling, and regenerative therapies.

\section{Cardiac tissue engineering for drug discovery and disease modeling}

It is well known that cardiovascular safety liabilities remain a major cause of drug attrition, resulting in early termination of pipeline candidates or withdrawal of marketed drugs ${ }^{192}$. Commonly used platforms to assess safety include small animal models, primarily mice, and ion-channel overexpressing cell lines ${ }^{193,194}$, which fail to truly predict human drug response effects (proarrhythmic, cardiotonic, and cardiotoxic) due to differences in cardiovascular physiopathology. Thus, cardiac TE-based solutions, such as spheroids $^{120,195}$, microtissues ${ }^{63,124}$, and "heart-on-a-chip"/microfluidic devices ${ }^{175,196,197}$, have been emerging as advanced culture systems, capable of improving the physiologic relevance over traditional $2 \mathrm{D}$ in vitro culture platforms. In these systems, hPSCCMs have shown higher predictive insight regarding drug mechanistic (e.g., inotropic response ${ }^{101,175,198}$ and ECC blockage $^{101,175}$ ) and toxic (e.g., loss of contractile function ${ }^{198}$ and apoptosis ${ }^{195}$ ) effects. Nonetheless, additional efforts are required to improve the reliability of these systems. For instance, a recent study demonstrated that linsitinib, a promising drug candidate to 


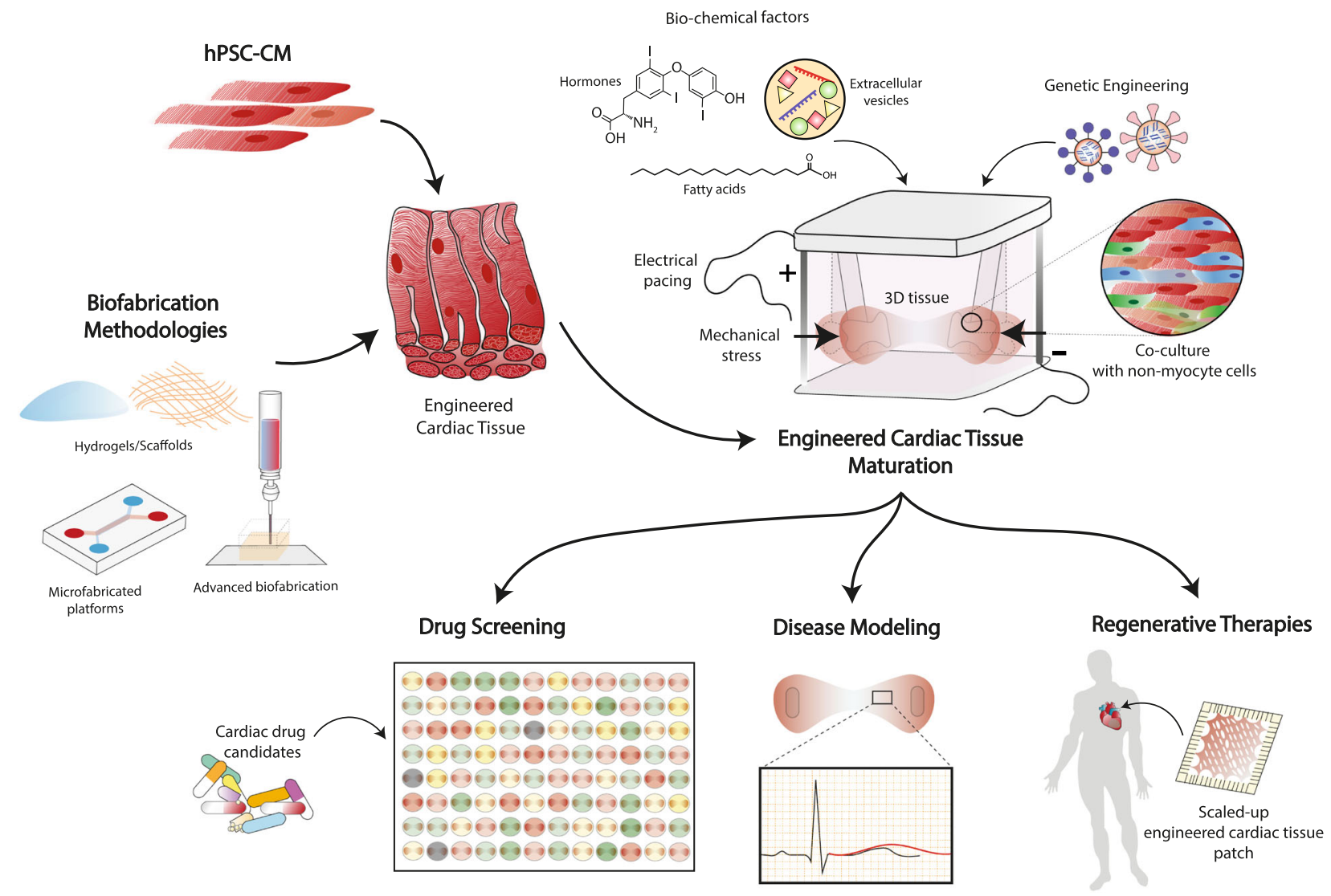

Fig. 4 Streamlining engineered cardiac tissues for precision medicine applications. Tissue engineering expertise enables the generation of physiologically relevant cardiac tissues grown from human pluripotent stem cell-derived cardiomyocytes (hPSC-CMs). Designing a proper maturation strategy will allow the maturation of hPSC-derived cardiac tissues to an adult-like phenotype, which can later on be used for patient-specific drug screening, disease modeling, and regenerative therapies.

treat Ewing sarcoma, significantly reduced tumor viability and cardiac function when both of these engineered tissues were cultured separately under perfusion in an organ-on-a-chip system. However, when they were cultured in an integrated way in the same platform, linsitinib showed poor tumor response and less cardiotoxicity, which was in agreement with the unfortunate clinical trial outcome of this drug ${ }^{199}$. Testing the targeted or offtargeted effect of new cardiovascular drugs will require advancing the physiological relevance in which cardiac engineered tissues are cultured. In addition, the complexity of engineered cardiac tissues is still incompatible with a rapid screen of large libraries of compounds, despite their smaller size in relation to a whole tissue/ organ. Although some platforms have shown the ability to be scaled-down 124,200,201, high-throughput culture modes and reproducible assessment of tissue functionality are still lacking ${ }^{202}$.

Expediting drug development will further require reliable models for a better understanding of disease mechanisms that guide target drug validation/discovery. In this context, hPSCs, as stated above, have emerged as attractive tools and a preferred source for obtaining cells for in vitro models, since these cells can be derived from patients using minimally invasive procedures, and preserve the genetic and phenotypic characteristics of the donors, including pathological traits responsible for disease onset and progression ${ }^{203,204}$. Several studies have been using 2D hiPSC-CM systems to investigate a plethora of genetic mutations associated with genetic heart diseases, including the main inherited cardiomyopathies (i.e., familial hypertrophic cardiomyopathy ${ }^{205}$, dilated cardiomyopathy ${ }^{203,206}$, arrhythmogenic cardiomyopathy ${ }^{207}$, and left ventricular noncompaction cardiomyopathy ${ }^{208}$ ) and channelopathies (e.g., type-2 long-QT syndrome 209 and
Timothy syndrome ${ }^{210}$ ). Others are modeling diseases without a known disease-causing genetic variant, such as idiosyncratic druginduced cardiotoxicity ${ }^{204,211,212}$. In parallel with the use of patientderived hPSCs, recent advances in targeted gene editing using the CRISPR-Cas9 systems have been performed on hPSCs ${ }^{213-215}$. Employing this technology, any wild-type cell can be altered to harbor a specific mutation, and a disease-causing mutation can be corrected in patient-derived cells, generating an isogenic control cell line. To the best of our knowledge, there are 13 completed or active clinical studies (www.clinicaltrials.gov) with the goal of generating patient-specific CMs to study the molecular mechanisms involved in several cardiac diseases, such as genetic and hypertrophic cardiomyopathies, inherited arrhythmias and valvulopathies, and cardiac fibrosis, among others (Table 1).

Notwithstanding, the use of engineered cardiac tissues as disease models is a relatively new concept. hPSC-CM tissue platforms harboring genetic mutations characteristic of hypertrophic cardiomyopathy ${ }^{216}$, dilated cardiomyopathy ${ }^{217}$, and mitochondrial cardiomyopathy of Barth syndrome ${ }^{218}$ have reported abnormalities in cardiac function congruent with a pathological phenotype. More recently, chamber-specific arrhythmia was demonstrated in a ringshaped cardiac tissue containing both ventricular and atrial hPSC$\mathrm{CMs}^{219}$. Manipulating engineered tissue microenvironment to induce pathological changes, such as $\mathrm{CM}$ remodeling of myocardium failure ${ }^{220}$, excessive ECM deposition of cardiac fibrosis ${ }^{221}$, and thrombus formation ${ }^{222}$, could similarly provide invaluable insights into mechanistic studies or predict patient-specific disease progression. Even though promising, disease-carrying hPSC-CMs still present an immature phenotype, thus possibly confounding identified disease hallmarks and identified mechanisms. For 
Table 1. Human clinical studies for the generation of disease and/or patient-specific models of cardiac diseases (Trial Identifier: www.clinicaltrials. gov).

\begin{tabular}{|c|c|c|c|}
\hline Study title & Condition(s) & Study type & Trial identifier \\
\hline $\begin{array}{l}\text { Modeling and pharmacological targeting of genetic } \\
\text { cardiomyopathy in children via iPSC-CM (DMDstem) }\end{array}$ & Familial cardiomyopathy & Interventional & NCT03696628 \\
\hline Evaluating cardiovascular phenotypes using iPSC & Coronary artery disease & Observational & NCT01517425 \\
\hline $\begin{array}{l}\text { Generation of Marfan syndrome and Fontan } \\
\text { cardiovascular models using patient-specific iPSC }\end{array}$ & Marfan's syndrome & Observational & NCT02815072 \\
\hline $\begin{array}{l}\text { Translational approaches to septic cardiomyopathy } \\
\text { (TASC01) }\end{array}$ & Cardiomyopathies, sepsis, septic shock & Observational & NCT03252613 \\
\hline $\begin{array}{l}\text { CIQTP prolongation: role and mechanism in sudden } \\
\text { cardiac death (IQARE-SCD) }\end{array}$ & Sudden cardiac death & Observational & NCT03387072 \\
\hline $\begin{array}{l}\text { Early MRI detection of myocardial deterioration as a } \\
\text { preventive, disease staging, and prognostic biomarker in } \\
\text { insulin resistance }\end{array}$ & $\begin{array}{l}\text { Cardiomyopathies; insulin resistance; non-ischemic } \\
\text { cardiomyopathy; cardiac fibrosis; diabetes }\end{array}$ & Observational & NCT03509441 \\
\hline $\begin{array}{l}\text { Characterization of patients with uncommon } \\
\text { presentations and/or uncommon diseases associated } \\
\text { with the cardiovascular system }\end{array}$ & $\begin{array}{l}\text { Cardiomyopathy; Li-Fraumeni syndrome; Parkinson's } \\
\text { disease; atherosclerosis; cardiovascular capacity }\end{array}$ & Observational & NCT01143454 \\
\hline UTHealth Turner syndrome research registry & Turner syndrome & Observational & NCT03185702 \\
\hline
\end{tabular}

instance, genetically engineered hPSC-CM microtissues carrying mutations that truncate the sarcomere protein titin, a known common cause of dilated cardiomyopathy, exhibited reduced contractility and decreased sarcomere length, among other abnormalities $^{217}$, none of which were detected in adult samples ${ }^{223}$. As a matter of fact, cardiac tissue structural maturity imposed by intensive electrical training was necessary to model pathological ventricular hypertrophy ${ }^{101,175}$. Even with their current limitations, the identified pathomechanisms in diseased engineered cardiac tissues can, in some cases, be translated to patient care in a personalized way. Recent research found out diltiazem, an L-type calcium channel blocker, was effective to correct disease-specific cardiac abnormalities in patient-derived engineered muscle strips with a rare form of hypertrophic cardiomyopathy. Diltiazem was further translated into patient care and ameliorated the prolonged QTc interval after 1 month of treatment ${ }^{224}$. These encouraging results give hope that emerging TE platforms could be extremely useful to reverse the growing burden associated with cardiovascular disorders.

\section{Cardiac tissue engineering for heart regenerative therapy}

Regenerative cardiac cell therapy broadly aims to achieve two complementary goals: (i) the direct cell replacement of the injured myocardium with contractile CMs and (ii) paracrine modulation of endogenous repair processes, such as angiogenesis, inflammation, apoptosis, and fibrosis, with both contractile and noncontractile cells $^{14,225,226}$. To the best of our knowledge, there are over 40 completed clinical trials exploring the therapeutic potential of cellbased therapies for cardiac regeneration and repair (www. clinicaltrials.gov). Pioneer studies tested non-cardiomyogenic cell populations that can easily be prepared for clinical applications, namely bone marrow-derived mononuclear cells (BM-MNCs) or MSCs, which in spite of their limited regenerative potential, may stimulate endogenous regenerative responses ${ }^{225,227}$. Secondgeneration cell candidates require a more refined isolation process, and ex vivo amplification procedures, but have higher regenerative potential, such as cardiac-derived progenitor cells and hPSC-CMs ${ }^{225,227}$. However, the clinical outcome of most of these studies remains insufficient, with evidence suggesting the major common obstacles to be the low engraftment and survival rate of transplanted cells in the host tissue, and the scarcity of endogenous cells with repair capacity ${ }^{225,228-230}$.

Cardiac TE has proven to be an effective method to improve the delivery, engraftment, and differentiation of stem cells in injured hearts, and therefore has gained much attention in recent years. Cardiac tissue surrogates developed to date are typically engineered cardiac muscle strips ${ }^{231-233}$, epicardial patches ${ }^{126,234-236}$, or cardiogenic scaffolds ${ }^{237}$, even though other strategies can be employed $^{238-243}$, such as acellular hydrogels to improve infarct mechanical integrity ${ }^{241-243}$. A summary of relevant cardiac TE preclinical studies in animal models is highlighted in Table 2 . When transplanted into infarcted or defected animal hearts, hPSC-CMbased tissues led to longer-term cell retentions ${ }^{231}$, progressively matured in vivo ${ }^{231}$, (imperfectly) electrically coupled with the host tissue $^{233}$, reversed infarction-associated changes (e.g., ameliorated cardiac remodeling by decreasing the amount of fibrosis ${ }^{241-243}$ enhanced vascularization ${ }^{126,233,242}$ ), and overall improved left ventricular function ${ }^{231,240,243}$. Recent advances have allowed rapid fabrication of clinically relevant sized human cardiac tissues able to maintain functionality preimplantation ${ }^{126,234}$, thus being suitable for large animal preclinical studies and future clinical applications. 


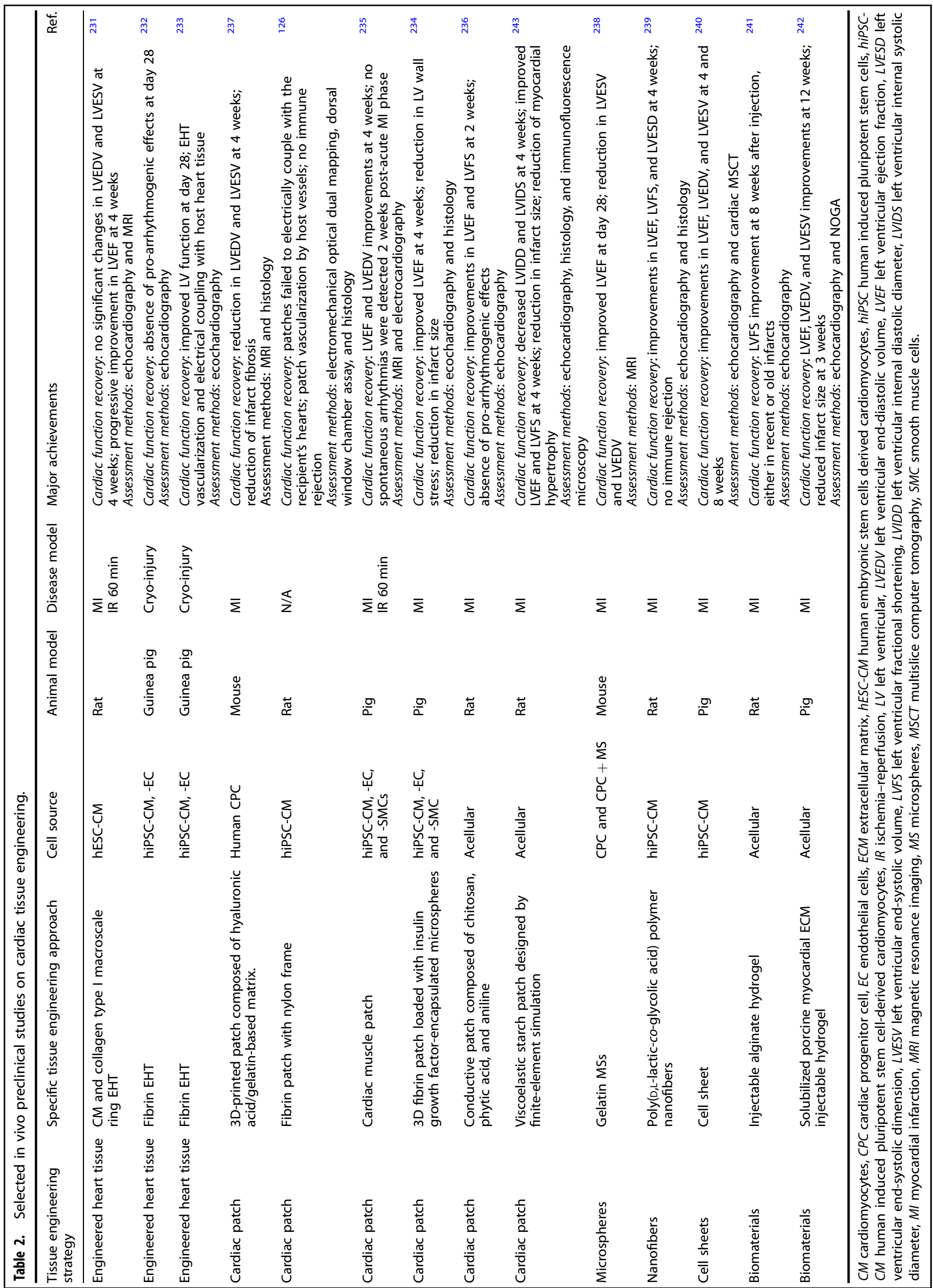


Table 3. Human clinical trial for cardiac tissue engineering (Trial Identifier: www.clinicaltrials.gov).

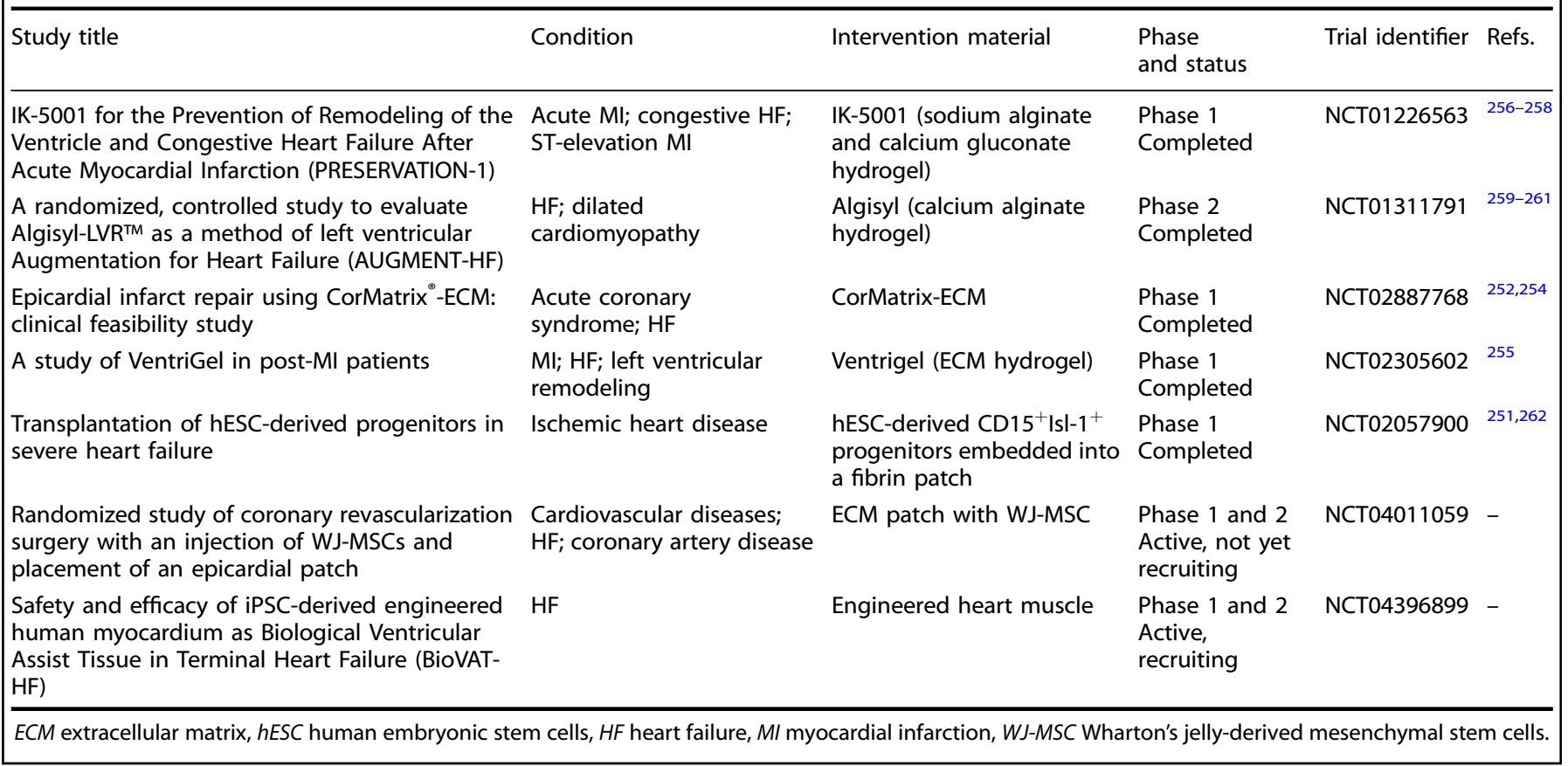

Among relevant risks that have to be taken into account in these therapies are the development of arrhythmias ${ }^{244,245}$, uncontrolled proliferation, and unwanted differentiation of cells that may lead to tumor formation in the case of hPSC-based therapies ${ }^{245-248}$, or to calcification of the host myocardium by MSCs or BM-MNCs ${ }^{247,249}$. Another potential issue is the development of an adverse immune response against the cells and materials employed in the constructs ${ }^{250}$. Designing tissues with a more controlled cell composition, defined architectural structure and developing strategies favoring muscular maturation before implantation could further improve their regenerative capability.

Despite showing promising outcomes in preclinical settings, the potential use of cardiac TE-based therapies at a clinical level is still in its infancy. Preliminary data from first-in-man clinical trials are providing evidence that cardiac TE-based therapies are generally safe $^{251,252}$, but do not provide relevant functional benefits. We are aware of five completed and two active phase $1 / 2$ studies mainly aiming at evaluating the safety and feasibility of cardiac TE therapeutic interventions, but also collecting preliminary efficacy data (Table 3).

The first studies being conducted are focused on the hypothesis that biomaterials could act as an internal wall support to increase infarct wall thickness, thereby improving cardiac function. Results from PRESERVATION I and AUGMENT-HF trials were, however, disappointing, with minor improvements in symptoms and clinical status for patients with advanced heart failure (HF), but no significant changes in left ventricle (LV) remodeling. Aiming to provide not only mechanical support for the prevention of infarct expansion but also to restore ECM homeostasis and architecture, other strategies relied on the epicardial or myocardial application of ECM patches or hydrogels. In particular, CorMatrix -ECM patches, derived from porcine small intestinal submucosa (SISECM), were applied on the epicardium of patients undergoing coronary artery bypass grafting, after an acute ischemic event. Porcine SIS-ECM has been used for over 20 years in a range of cardiovascular applications, but of all the commercially available SIS-ECM products, CorMatrix is the only medical device with clearance, even though there are few reports regarding its use for myocardial regeneration after acute myocardial infarction in humans ${ }^{253}$. No results for this study have been published so far; however, based on preliminary data obtained in rodent and porcine models, the patches are expected to not only prevent infarct expansion and LV dilation but also restore ECM homeostasis and architecture ${ }^{252,254}$. Another recently completed study evaluated the effect of VentriGel, a myocardium-specific ECM hydrogel, on patients in a 60- to 3-year window since their first acute ST-elevation myocardial infarction. Using a minimally invasive procedure, the gel was injected using a NOGA-MyoStar catheter, allowing for an intramyocardial delivery of the therapeutic gel on mapped infarcted areas. Overall, VentriGel showed encouraging safety results, with no deaths or patients who discontinued from the study, nor adverse events reported as a direct consequence of the treatment ${ }^{255}$. Although efficacy was not a primary outcome, there were suggestions of improvements, including in the 6-min walk test distance and decreases in New York Heart Association functional classification, across the entire cohort of patients ${ }^{255}$. Improvements in LV remodeling data, viable mass, and ventricular natriuretic peptide levels were mainly observed in patients who had a myocardial infarction $>12$ months before treatment ${ }^{255}$

A distinctive approach has been the delivery of clinical-grade hESC-derived cardiovascular progenitor cells in fibrin patches for the treatment of severe ischemic left ventricular dysfunction ${ }^{251}$. Compared with biomaterial-only methods, cell patches showed equal safety profiles, with no complications that could be specifically ascribed to the cellular graft, namely, no detection of teratomas that could be developed from residual pluripotent cells, no occurrence of arrhythmias, and no major alloimmunization events. Still, following a similar trend as previous strategies, no astonishing results were obtained in terms of efficacy, although all patients were symptomatically improved. A newly posted study aims at determining the safety of Wharton's jelly-derived MSCs from human umbilical cord seeded on ECM patches, and also test its efficacy to promote neomyogenesis in patients with previous myocardial infarction. Another active and recently recruiting promising clinical trial will evaluate the efficacy of an engineered 
heart muscle (EHM) to sustainably remuscularize and improve myocardial performance in patients with advanced HF with reduced ejection fraction ( $\leq 35 \%)$. The EHM, a construct generated from hiPSC-CMs and stromal cells in a bovine collagen type I hydrogel, will be the first of its kind to be tested in humans.

Despite the marginally positive outcome of all these clinical trials, this does not necessarily imply a lack of therapeutic value and may result instead from an inadequate choice of biomaterial and/or cell type or inaccuracies in dosing and/or delivery. A better understanding underlying the mechanism of action of these advanced medicinal products may contribute to further improvements down the road.

\section{CONCLUSIONS}

To date, inducing maturation of hPSC-CMs is a challenge in the field, limiting their use in clinical settings. Considerable progress has been achieved by continuously seeking innovative ways to improve hPSC-CM maturation state, and, currently, it is already possible to obtain cardiac tissue with adult-like gene signatures, remarkable ultrastructural organization, functional contractile machinery, and improved electrophysiology. Even though in vitro maturation may not follow the in vivo paradigm, identifying the spatio-temporal mechanisms dictating tissue development and designing a combinatorial strategy capable of addressing such complexity might be required to engineer fully matured biomimetic cardiac tissues. Given the interdisciplinarity of TE, from stem cell biology to stem cell bioprocessing, material science, and advanced biofabrication, taking advantage of this knowledge would be beneficial to grow physiologically relevant cardiac tissues in vitro. Recreating cardiac tissue complexity is still challenging, limiting the size of the engineered tissue possible to be obtained, and thus redirecting their application towards other areas rather than the clinical transplantation arena. Benchmarked "on-a-chip" engineered cardiac tissues, although promising, still need to show standardization and high-throughput compatibility to contribute to more reliable results than commonly used platforms, as well as reduction of costs to expedite drug development. Successful attempts at scaling up engineered cardiac tissues have made it possible to investigate their regenerative efficacy in large animal studies, and the results of pioneering TE-based clinical trials are eagerly awaited. While there are still few clinical trials, these have been an important step in the ability to engineer artificial constructs that mimic some of the important compositional, architectural, and functional properties of human cardiac tissues. Even if it is not unrealistic to foresee a translation of engineered cardiac tissues for in vitro clinical studies, their potential to remuscularize failing hearts and integrate the host tissue without adverse effects, despite a better retention, is still uncertain, and the causes associated with mild improvements unknown. Nonetheless, advances in the field of cardiac TE have undoubtedly imposed a new paradigm in cardiovascular science, paving the way one day for restoring or even replacing damaged hearts.

Received: 8 December 2020; Accepted: 10 May 2021; Published online: 01 June 2021

\section{REFERENCES}

1. Bloom, D. E. et al. The Global Economic Burden of Non-communicable Diseases. Geneva: World Economic Forum (2011).

2. Roth, G. A. et al. Global, regional, and national burden of cardiovascular diseases for 10 causes, 1990 to 2015. J. Am. Coll. Cardiol. 70, 1-25 (2017).

3. Jessup, M. \& Brozena, S. Heart failure. N. Engl. J. Med. 348, 2007-2018 (2003).

4. Bergmann, O. et al. Evidence for cardiomyocyte renewal in humans. Science 324, 98-102 (2009).
5. Menasché, P. et al. The myoblast autologous grafting in ischemic cardiomyopathy (MAGIC) trial: first randomized placebo-controlled study of myoblast transplantation. Circulation 117, 1189-1200 (2008).

6. Assmus, B. et al. Clinical outcome 2 years after intracoronary administration of bone marrow-derived progenitor cells in acute myocardial infarction. Circ. Heart Fail. 3, 89-96 (2010).

7. Strauer, B. E., Yousef, M. \& Schannwell, C. M. The acute and long-term effects of intracoronary stem cell transplantation in 191 patients with chronic heARt failure: the STAR-heart study. Eur. J. Heart Fail. 12, 721-729 (2010).

8. Menasché, P. et al. Human embryonic stem cell-derived cardiac progenitors for severe heart failure treatment: first clinical case report. Eur. Heart J. 36, 2011-2017 (2015).

9. Kastrup, J. et al. Direct intramyocardial plasmid vascular endothelial growth factor-A 165 gene therapy in patients with stable severe angina pectoris: a randomized double-blind placebo-controlled study: the Euroinject One trial. J. Am. Coll. Cardiol. 45, 982-988 (2005).

10. Zohlnhofer, D. et al. Stem cell mobilization by granulocyte colony-stimulating factor in patients. JAMA 295, 1003-1010 (2006).

11. Gao, R. et al. A phase II, randomized, double-blind, multicenter, based on standard therapy, placebo-controlled study of the efficacy and safety of recombinant human neuregulin-1 in patients with chronic heart failure. J. Am. Coll. Cardiol. 55, 1907-1914 (2010).

12. Chien, K. R. et al. Regenerating the field of cardiovascular cell therapy. Nat. Biotechnol. 37, 232-237 (2019).

13. Khademhosseini, A. \& Langer, R. A decade of progress in tissue engineering. Nat. Protoc. 11, 1775-1781 (2016).

14. Madonna, R. et al. ESC Working Group on Cellular Biology of the Heart Position paper for Cardiovascular Research: tissue engineering and cell-based therapies for cardiac repair in ischemic heart disease and heart failure. Cardiovasc. Res. 115, 488-500 (2019).

15. Zhao, Y. et al. Towards chamber specific heart-on-a-chip for drug testing applications. Adv. Drug Deliv. Rev. 165, 60-76 (2020).

16. Zhou, P. \& Pu, W. T. Recounting cardiac cellular composition. Circ. Res. 118, 368-370 (2016).

17. Litviňuková, M. et al. Cells of the adult human heart. Nature 588, 466-472 (2020).

18. Hescheler, J. et al. Morphological, biochemical, and electrophysiological characterization of a clonal cell (H9c2) line from rat heart. Circ. Res. 69, 1476-1486 (1991).

19. Claycomb, W. C. et al. HL-1 cells: a cardiac muscle cell line that contracts and retains phenotypic characteristics of the adult cardiomyocyte. Proc. Natl Acad. Sci. USA 95, 2979-2984 (1998).

20. Eschenhagen, T. et al. Three-dimensional reconstitution of embryonic cardiomyocytes in a collagen matrix: a new heart muscle model system. FASEB J. 11, 683-694 (1997).

21. Carrier, R. L. et al. Cardiac tissue engineering: cell seeding, cultivation parameters, and tissue construct characterization. Biotechnol. Bioeng. 64, 580-589 (1999).

22. Watson, S. A. et al. Preparation of viable adult ventricular myocardial slices from large and small mammals. Nat. Protoc. 12, 2623-2639 (2017).

23. Banyasz, T. et al. Transformation of adult rat cardiac myocytes in primary culture. Exp. Physiol. 93, 370-382 (2008).

24. Watson, S. A. et al. Biomimetic electromechanical stimulation to maintain adult myocardial slices in vitro. Nat. Commun. 10, 1-15 (2019).

25. Fischer, $C$. et al. Long-term functional and structural preservation of precisioncut human myocardium under continuous electromechanical stimulation in vitro. Nat. Commun. 10, 1-12 (2019).

26. Davidson, M. M. et al. Novel cell lines derived from adult human ventricular cardiomyocytes. J. Mol. Cell. Cardiol. 39, 133-147 (2005).

27. Thomson, J. A. et al. Embryonic stem cell lines derived from human blastocysts. Science 282, 1145-1147 (1998).

28. Takahashi, K. \& Yamanaka, S. Induction of pluripotent stem cells from mouse embryonic and adult fibroblast cultures by defined factors. Cell 126, 663-676 (2006).

29. Takahashi, K. et al. Induction of pluripotent stem cells from adult human fibroblasts by defined factors. Cell 131, 861-872 (2007).

30. Chen, I. Y., Matsa, E. \& Wu, J. C. Induced pluripotent stem cells: at the heart of cardiovascular precision medicine. Nat. Rev. Cardiol. 13, 333-349 (2016).

31. Inoue, H., Nagata, N., Kurokawa, H. \& Yamanaka, S. iPS cells: a game changer for future medicine. EMBO J. 33, 409-417 (2014).

32. Burridge, P. W. et al. Chemically defned generation of human cardiomyocytes. Nat. Methods 11, 855-860 (2014).

33. Ueno, $\mathrm{S}$. et al. Biphasic role for $\mathrm{Wnt} / \beta$-catenin signaling in cardiac specification in zebrafish and embryonic stem cells. Proc. Natl Acad. Sci. USA 104, 9685-9690 (2007). 
34. Lian, X. et al. Robust cardiomyocyte differentiation from human pluripotent stem cells via temporal modulation of canonical Wnt signaling. Proc. Natl Acad. Sci. USA 109, E1848-E1857 (2012).

35. Wang, H., Hao, J. \& Hong, C. C. Cardiac induction of embryonic stem cells by a small molecule inhibitor of Wnt/ $\beta$-catenin signaling. ACS Chem. Biol. 6, 192-197 (2011).

36. Lian, $X$. et al. Directed cardiomyocyte differentiation from human pluripotent stem cells by modulating $W n t / \beta$-catenin signaling under fully defined conditions. Nat. Protoc. 8, 162-175 (2013).

37. Squire, J. M. Architecture and function in the muscle sarcomere. Curr. Opin. Struct. Biol. 7, 247-257 (1997).

38. Gerdes, A. M. et al. Structural remodeling of cardiac myocytes in patients with ischemic cardiomyopathy. Circulation 86, 426-430 (1992).

39. Mollova, M. et al. Cardiomyocyte proliferation contributes to heart growth in young humans. Proc. Natl Acad. Sci. USA 110, 1446-1451 (2013)

40. Olivetti, G. et al. Aging, cardiac hypertrophy and ischemic cardiomyopathy do not affect the proportion of mononucleated and multinucleated myocytes in the human heart. J. Mol. Cell. Cardiol. 28, 1463-1477 (1996).

41. Wei, S. et al. T-tubule remodeling during transition from hypertrophy to heart failure. Circ. Res. 107, 520-531 (2010).

42. Kaprielian, R. R., Stevenson, S., Rothery, S. M., Cullen, M. J. \& Severs, N. J. Distinct patterns of dystrophin organization in myocyte sarcolemma and transverse tubules of normal and diseased human myocardium. Circulation 101, 2586-2594 (2000)

43. Pinali, C., Bennett, H., Davenport, J. B., Trafford, A. W. \& Kitmitto, A. Threedimensional reconstruction of cardiac sarcoplasmic reticulum reveals a continuous network linking transverse-tubules: this organization is perturbed in heart failure. Circ. Res. 113, 1219-1230 (2013).

44. Porter, G. A. et al. Bioenergetics, mitochondria, and cardiac myocyte differentiation. Prog. Pediatr. Cardiol. 31, 75-81 (2011).

45. Saffitz, J. E., Kanter, H. L., Green, K. G., Tolley, T. K. \& Beyer, E. C. Tissue-specific determinants of anisotropic conduction velocity in canine atrial and ventricular myocardium. Circ. Res. 74, 1065-1070 (1994).

46. Peters, N. S. et al. Spatiotemporal relation between gap junctions and fascia adherens junctions during postnatal development of human ventricular myocardium. Circulation 90, 713-725 (1994).

47. Lundy, S. D., Zhu, W. Z., Regnier, M. \& Laflamme, M. A. Structural and functional maturation of cardiomyocytes derived from human pluripotent stem cells. Stem Cells Dev. 22, 1991-2002 (2013).

48. Snir, M. et al. Assessment of the ultrastructural and proliferative properties of human embryonic stem cell-derived cardiomyocytes. Am. J. Physiol. Heart Circ. Physiol. 285, 2355-2363 (2003).

49. Vreeker, A. et al. Assembly of the cardiac intercalated disk during pre- and postnatal development of the human heart. PLoS ONE 9, e94722 (2014).

50. Liu, J., Fu, J. D., Siu, C. W. \& Li, R. A. Functional sarcoplasmic reticulum for calcium handling of human embryonic stem cell-derived cardiomyocytes: insights for driven maturation. Stem Cells 25, 3038-3044 (2007).

51. Lieu, D. K. et al. Absence of transverse tubules contributes to non-uniform $\mathrm{Ca} 2+$ wavefronts in mouse and human embryonic stem cell-derived cardiomyocytes. Stem Cells Dev. 18, 1493-1500 (2009).

52. Dai, D. F., Danoviz, M. E., Wiczer, B., Laflamme, M. A. \& Tian, R. Mitochondrial maturation in human pluripotent stem cell derived cardiomyocytes. Stem Cells Int. 2017, 5153625 (2017).

53. Schaaf, $S$. et al. Human engineered heart tissue as a versatile tool in basic research and preclinical toxicology. PLoS ONE 6, e26397 (2011).

54. Sasaki, D. et al. Contractile force measurement of human induced pluripotent stem cell-derived cardiac cell sheet-tissue. PLOS ONE 13, e0198026 (2018).

55. Lopaschuk, G. D. \& Jaswal, J. S. Energy metabolic phenotype of the cardiomyocyte during development, differentiation, and postnatal maturation. J. Cardiovasc. Pharmacol. 56, 130-140 (2010).

56. Chung, S. et al. Mitochondrial oxidative metabolism is required for the cardiac differentiation of stem cells. Nat. Clin. Pract. Cardiovasc. Med. 4, 60-67 (2007).

57. Kolwicz, S. C., Purohit, S. \& Tian, R. Cardiac metabolism and its interactions with contraction, growth, and survival of cardiomyocytes. Circ. Res. 113, 603-616 (2013).

58. Van Der Velden, J. et al. Isometric tension development and its calcium sensitivity in skinned myocyte-sized preparations from different regions of the human heart. Cardiovasc. Res. 42, 706-719 (1999).

59. Hasenfuss, G. et al. Energetics of isometric force development in control and volume-overload human myocardium. Comparison with animal species. Circ. Res. 68, 836-846 (1991).

60. Mulieri, L. A., Hasenfuss, G., Leavitt, B., Allen, P. D. \& Alpert, N. R. Altered myocardial force-frequency relation in human heart failure. Circulation $\mathbf{8 5}$ 1743-1750 (1992).

61. Pieske, B. et al. Diminished post-rest potentiation of contractile force in human dilated cardiomyopathy: functional evidence for alterations in intracellular $\mathrm{Ca} 2+$ handling. J. Clin. Invest. 98, 764-776 (1996).
62. Mannhardt, I. et al. Human engineered heart tissue: analysis of contractile force. Stem Cell Rep. 7, 29-42 (2016).

63. Huebsch, N. et al. Miniaturized iPS-cell-derived cardiac muscles for physiologically relevant drug response analyses. Sci. Rep. 6, 1-12 (2016).

64. Antzelevitch, C. \& Burashnikov, A. Overview of basic mechanisms of cardiac arrhythmia. Card. Electrophysiol. Clin. 3, 23-45 (2011).

65. Dangman, K. H. et al. Electrophysiologic characteristics of human ventricular and Purkinje fibers. Circulation 65, 362-368 (1982).

66. Satin, J. et al. Mechanism of spontaneous excitability in human embryonic stem cell derived cardiomyocytes. J. Physiol. 559, 479-496 (2004).

67. Mummery, C. et al. Differentiation of human embryonic stem cells to cardiomyocytes: role of coculture with visceral endoderm-like cells. Circulation 107, 2733-2740 (2003).

68. Ribeiro, M. C. et al. Functional maturation of human pluripotent stem cell derived cardiomyocytes invitro - correlation between contraction force andelectrophysiology. Biomaterials 51, 138-150 (2015).

69. Scheel, O. et al. Action potential characterization of human induced pluripotent stem cell-derived cardiomyocytes using automated patch-clamp technology. Assay Drug Dev. Technol. 12, 457-469 (2014).

70. Prajapati, C., Pölönen, R. P. \& Aalto-Setälä, K. Simultaneous recordings of action potentials and calcium transients from human induced pluripotent stem cell derived cardiomyocytes. Biol. Open 7, bio035030 (2018)

71. Bers, D. M. Cardiac excitation-contraction coupling. Nature 415, 198-205 (2002).

72. Zhang, G. Q., Wei, H., Lu, J., Wong, P. \& Shim, W. Identification and characterization of calcium sparks in cardiomyocytes derived from human induced pluripotent stem cells. PLoS ONE 8, e55266 (2013).

73. Riedel, M. et al. Functional and pharmacological analysis of cardiomyocytes differentiated from human peripheral blood mononuclear-derived pluripotent stem cells. Stem Cell Rep. 3, 131-141 (2014).

74. Thompson, S. A. et al. Engraftment of human embryonic stem cell derived cardiomyocytes improves conduction in an arrhythmogenic in vitro model. J. Mol. Cell. Cardiol. 53, 15-23 (2012).

75. Trautwein, W., Kassebaum, D. G., Nelson, R. M. \& Hecht, H. H. Electrophysiological study of human heart muscle. Circ. Res. 10, 306-312 (1962).

76. Kupersmith, J., Krongrad, E. \& Waldo, A. L. Conduction intervals and conduction velocity in the human cardiac conduction system. Studies during open-heart surgery. Circulation 47, 776-785 (1973).

77. Synnergren, J., Améen, C., Jansson, A. \& Sartipy, P. Global transcriptional profiling reveals similarities and differences between human stem cell-derived cardiomyocyte clusters and heart tissue. Physiol. Genomics 44, 245-258 (2012).

78. Scuderi, G. J. \& Butcher, J. Naturally engineered maturation of cardiomyocytes. Front. Cell Dev. Biol. 5, 50 (2017).

79. Kannan, S. \& Kwon, C. Regulation of cardiomyocyte maturation during critical perinatal window. J. Physiol. 598, 2941-2956 (2020).

80. Kamakura, T. et al. Ultrastructural maturation of human-induced pluripotent stem cell-derived cardiomyocytes in a long-term culture. Circ. J. 77, 1307-1314 (2013).

81. Ebert, A. et al. Proteasome-dependent regulation of distinct metabolic states during long-term culture of human ipsc-derived cardiomyocytes. Circ. Res. 125 90-103 (2019).

82. Shiba, Y. et al. Allogeneic transplantation of iPS cell-derived cardiomyocytes regenerates primate hearts. Nature 538, 388-391 (2016).

83. Kadota, S., Pabon, L., Reinecke, H. \& Murry, C. E. In vivo maturation of human induced pluripotent stem cell-derived cardiomyocytes in neonatal and adult rat hearts. Stem Cell Rep. 8, 278-289 (2017).

84. Radisic, M. et al. Functional assembly of engineered myocardium by electrical stimulation of cardiac myocytes cultured on scaffolds. Proc. Natl Acad. Sci. USA 101, 18129-18134 (2004).

85. Tandon, N. et al. Electrical stimulation systems for cardiac tissue engineering. Nat. Protoc. 4, 155-173 (2009).

86. Sathaye, A., Bursac, N., Sheehy, S. \& Tung, L. Electrical pacing counteracts intrinsic shortening of action potential duration of neonatal rat ventricular cells in culture. J. Mol. Cell. Cardiol. 41, 633-641 (2006).

87. Godier-Furnémont, A. F. G. et al. Physiologic force-frequency response in engineered heart muscle by electromechanical stimulation. Biomaterials $\mathbf{6 0}$, 82-91 (2015).

88. Kroll, $\mathrm{K}$. et al. Electro-mechanical conditioning of human iPSC-derived cardiomyocytes for translational research. Prog. Biophys. Mol. Biol. 130, 212-222 (2017).

89. Nunes, S. S. et al. Biowire: a platform for maturation of human pluripotent stem cell-derived cardiomyocytes. Nat. Methods 10, 781-787 (2013).

90. Zimmermann, W. H. et al. Tissue engineering of a differentiated cardiac muscle construct. Circ. Res. 90, 223-230 (2002).

91. Hansen, A. et al. Development of a drug screening platform based on engineered heart tissue. Circ. Res. 107, 35-44 (2010).

92. Jackman, C. P., Carlson, A. L. \& Bursac, N. Dynamic culture yields engineered myocardium with near-adult functional output. Biomaterials 111, 66-79 (2016). 
93. Breckwoldt, K. et al. Differentiation of cardiomyocytes and generation of human engineered heart tissue. Nat. Protoc. 12, 1177-1197 (2017).

94. Ulmer, B. M. et al. Contractile work contributes to maturation of energy metabolism in hiPSC-derived cardiomyocytes. Stem Cell Rep. 10, 834-847 (2018).

95. Uzun, A. U. et al. $\mathrm{Ca}^{2+}$-currents in human induced pluripotent stem cell-derived cardiomyocytes effects of two different culture conditions. Front. Pharmacol. 7, 300 (2016).

96. Leonard, A. et al. Afterload promotes maturation of human induced pluripotent stem cell derived cardiomyocytes in engineered heart tissues. J. Mol. Cell. Cardiol. 118, 147-158 (2018).

97. Abilez, O. J. et al. Passive stretch induces structural and functional maturation of engineered heart muscle as predicted by computational modeling. Stem Cells 36, 265-277 (2018).

98. Rogers, A. J., Fast, V. G. \& Sethu, P. Biomimetic cardiac tissue model enables the adaption of human induced pluripotent stem cell cardiomyocytes to physiological hemodynamic loads. Anal. Chem. 88, 9862-9868 (2016).

99. Hirt, M. N. et al. Functional improvement and maturation of rat and human engineered heart tissue by chronic electrical stimulation. J. Mol. Cell. Cardiol. 74, 151-161 (2014).

100. Ruan, J. L. et al. Mechanical stress conditioning and electrical stimulation promote contractility and force maturation of induced pluripotent stem cellderived human cardiac tissue. Circulation 134, 1557-1567 (2016).

101. Ronaldson-Bouchard, K. et al. Advanced maturation of human cardiac tissue grown from pluripotent stem cells. Nature 556, 239-243 (2018).

102. Ribeiro, A. J. S. et al. Contractility of single cardiomyocytes differentiated from pluripotent stem cells depends on physiological shape and substrate stiffness. Proc. Natl Acad. Sci. USA 112, 12705-12710 (2015).

103. Rodriguez, M. L. et al. Substrate stiffness, cell anisotropy, and cell-cell contact contribute to enhanced structural and calcium handling properties of human embryonic stem cell-derived cardiomyocytes. ACS Biomater. Sci. Eng. 5, 3876-3888 (2019).

104. Wang, P. Y., Yu, J., Lin, J. H. \& Tsai, W. B. Modulation of alignment, elongation and contraction of cardiomyocytes through a combination of nanotopography and rigidity of substrates. Acta Biomater. 7, 3285-3293 (2011).

105. Feaster, T. K. et al. Matrigel mattress: a method for the generation of single contracting human-induced pluripotent stem cell-derived cardiomyocytes. Circ. Res. 117, 995-1000 (2015).

106. Papadaki, M. et al. Tissue engineering of functional cardiac muscle: molecular, structural, and electrophysiological studies. Am. J. Physiol. Heart Circ. Physiol. 280, 168-178 (2001).

107. Chen, Y. et al. Engineering a freestanding biomimetic cardiac patch using biodegradable poly(lactic-co-glycolic acid) (PLGA) and human embryonic stem cellderived ventricular cardiomyocytes (hESC-VCMs). Macromol. Biosci. 15, 426-436 (2015).

108. Jongpaiboonkit, L. et al. An adaptable hydrogel array format for 3-dimensional cell culture and analysis. Biomaterials 29, 3346-3356 (2008).

109. Chun, Y. W. et al. Combinatorial polymer matrices enhance in vitro maturation of human induced pluripotent stem cell-derived cardiomyocytes. Biomaterials 67, 52-64 (2015)

110. Feinberg, A. W. et al. Muscular thin films for building actuators and powering devices. Science 317, 1366-1370 (2007).

111. Herron, T. J. et al. Extracellular matrix-mediated maturation of human pluripotent stem cell-derived cardiac monolayer structure and electrophysiological function. Circ. Arrhythmia Electrophysiol. 9, e003638 (2016).

112. Fong, A. H. et al. Three-dimensional adult cardiac extracellular matrix promotes maturation of human induced pluripotent stem cell-derived cardiomyocytes. Tissue Eng. Part A 22, 1016-1025 (2016).

113. Garreta, E. et al. Myocardial commitment from human pluripotent stem cells: rapid production of human heart grafts. Biomaterials 98, 64-78 (2016).

114. Guyette, J. P. et al. Bioengineering human myocardium on native extracellular matrix. Circ. Res. 118, 56-72 (2016).

115. Tian, Y. \& Morrisey, E. E. Importance of myocyte-nonmyocyte interactions in cardiac development and disease. Circ. Res. 110, 1023-1034 (2012).

116. Abecasis, B. et al. Unveiling the molecular crosstalk in a human induced pluripotent stem cell-derived cardiac model. Biotechnol. Bioeng. 116, 1245-1252 (2019).

117. Dunn, K. K. et al. Coculture of endothelial cells with human pluripotent stem cell-derived cardiac progenitors reveals a differentiation stage-specific enhancement of cardiomyocyte maturation. Biotechnol. J. 14, 1800725 (2019).

118. Burridge, P. W. et al. Multi-cellular interactions sustain long-term contractility of human pluripotent stem cell-derived cardiomyocytes. Am. J. Transl. Res. 6, 724-735 (2014).

119. Shimizu, T. et al. Fabrication of pulsatile cardiac tissue grafts using a novel 3dimensional cell sheet manipulation technique and temperature-responsive cell culture surfaces. Circ. Res. 90, e40-e48 (2002).
120. Correia, C. et al. 3D aggregate culture improves metabolic maturation of human pluripotent stem cell derived cardiomyocytes. Biotechnol. Bioeng. 115, 630-644 (2018).

121. Eng, G. et al. Autonomous beating rate adaptation in human stem cell-derived cardiomyocytes. Nat. Commun. 7, 1-10 (2016).

122. $\mathrm{Ma}$, Z. et al. Self-organizing human cardiac microchambers mediated by geometric confinement. Nat. Commun. 6, 1-10 (2015).

123. Goldfracht, l. et al. Engineered heart tissue models from hiPSC-derived cardiomyocytes and cardiac ECM for disease modeling and drug testing applications. Acta Biomater. 92, 145-159 (2019).

124. Boudou, T. et al. A microfabricated platform to measure and manipulate the mechanics of engineered cardiac microtissues. Tissue Eng. Part A 18, 910-919 (2012).

125. Bian, W., Badie, N., Himel, H. D. \& Bursac, N. Robust T-tubulation and maturation of cardiomyocytes using tissue-engineered epicardial mimetics. Biomaterials 35, 3819-3828 (2014).

126. Shadrin, I. Y. et al. Cardiopatch platform enables maturation and scale-up of human pluripotent stem cell-derived engineered heart tissues. Nat. Commun. 8 , 1-15 (2017).

127. Macqueen, L. A. et al. A tissue-engineered scale model of the heart ventricle. Nat. Biomed. Eng. 2, 930-941 (2018).

128. Thavandiran, N. et al. Design and formulation of functional pluripotent stem cell-derived cardiac microtissues. Proc. Natl Acad. Sci. USA 110, E4698-E4707 (2013).

129. Abecasis, B. et al. Toward a microencapsulated 3D hiPSC-derived in vitro cardiac microtissue for recapitulation of human heart microenvironment features. Front. Bioeng. Biotechnol. 8, 1163 (2020).

130. Giacomelli, E. et al. Human-iPSC-derived cardiac stromal cells enhance maturation in 3D cardiac microtissues and reveal non-cardiomyocyte contributions to heart disease. Cell Stem Cell 26, 862-879 (2020).

131. Ronaldson-Bouchard, K. et al. Engineering of human cardiac muscle electromechanically matured to an adult-like phenotype. Nat. Protoc. 14, 2781-2817 (2019).

132. Yang, X. et al. Fatty acids enhance the maturation of cardiomyocytes derived from human pluripotent stem cells. Stem Cell Rep. 13, 657-668 (2019).

133. $\mathrm{Hu}, \mathrm{D}$. et al. Metabolic maturation of human pluripotent stem cellderived cardiomyocytes by inhibition of HIF1a and LDHA. Circ. Res. 123, 1066-1079 (2018).

134. Correia, C. et al. Distinct carbon sources affect structural and functional maturation of cardiomyocytes derived from human pluripotent stem cells. Sci. Rep. 7, 1-17 (2017).

135. Li, M. et al. Thyroid hormone action in postnatal heart development. Stem Cell Res. 13, 582-591 (2014).

136. Yang, X. et al. Tri-iodo-l-thyronine promotes the maturation of human cardiomyocytes-derived from induced pluripotent stem cells. J. Mol. Cell. Cardiol. 72, 296-304 (2014).

137. Parikh, S. S. et al. Thyroid and glucocorticoid hormones promote functional Ttubule development in human-induced pluripotent stem cell-derived cardiomyocytes. Circ. Res. 121, 1323-1330 (2017).

138. Yoshida, S. et al. Maturation of human induced pluripotent stem cell-derived cardiomyocytes by soluble factors from human mesenchymal stem cells. Mol. Ther. 26, 2681-2695 (2018).

139. Deng, X. F., Rokosh, D. G. \& Simpson, P. C. Autonomous and growth factorinduced hypertrophy in cultured neonatal mouse cardiac myocytes: comparison with rat. Circ. Res. 87, 781-788 (2000).

140. Sucharov, C. C. et al. A $\beta 1$-adrenergic receptor CaM kinase II-dependent pathway mediates cardiac myocyte fetal gene induction. Am. J. Physiol. Heart Circ. Physiol. 291, 1299-1308 (2006).

141. Patrizio, M. et al. CAMP-mediated $\beta$-adrenergic signaling negatively regulates Gq-coupled receptor-mediated fetal gene response in cardiomyocytes. J. Mol. Cell. Cardiol. 45, 761-769 (2008).

142. Földes, G. et al. Modulation of human embryonic stem cell-derived cardiomyocyte growth: a testbed for studying human cardiac hypertrophy? J. Mol. Cell. Cardiol. 50, 367-376 (2011).

143. Lieu, D. K. et al. Mechanism-based facilitated maturation of human pluripotent stem cell-derived cardiomyocytes. Circ. Arrhythmia Electrophysiol. 6, 191-201 (2013).

144. Liu, J. et al. Facilitated maturation of $\mathrm{Ca}^{2+}$ handling properties of human embryonic stem cell-derived cardiomyocytes by calsequestrin expression. Am. J. Physiol. Cell Physiol. 297, 152-159 (2009).

145. Chow, M. Z. et al. Epigenetic regulation of the electrophysiological phenotype of human embryonic stem cell-derived ventricular cardiomyocytes: insights for driven maturation and hypertrophic growth. Stem Cells Dev. 22, 2678-2690 (2013).

146. Otsuji, T. G., Kurose, Y., Suemori, H., Tada, M. \& Nakatsuji, N. Dynamic link between histone $\mathrm{H} 3$ acetylation and an increase in the functional characteristics of human ESC/iPSC-derived cardiomyocytes. PLoS ONE 7, e45010 (2012). 
147. Biermann, M. et al. Epigenetic priming of human pluripotent stem cell-derived cardiac progenitor cells accelerates cardiomyocyte maturation. Stem Cells 37, 910-923 (2019).

148. Babiarz, J. E. et al. Determination of the human cardiomyocyte mRNA and miRNA differentiation network by fine-scale profiling. Stem Cells Dev. 21, 1956-1965 (2012).

149. Kumar, N. et al. Assessment of temporal functional changes and miRNA profiling of human iPSC-derived cardiomyocytes. Sci. Rep. 9, 1-16 (2019).

150. Kuppusamy, K. T. et al. Let-7 family of microRNA is required for maturation and adult-like metabolism in stem cell-derived cardiomyocytes. Proc. Natl Acad. Sci. USA 112, E2785-E2794 (2015).

151. Lu, T. Y. et al. Overexpression of microRNA-1 promotes cardiomyocyte commitment from human cardiovascular progenitors via suppressing WNT and FGF signaling pathways. J. Mol. Cell. Cardiol. 63, 146-154 (2013).

152. $\mathrm{Fu}, \mathrm{J}$. D. et al. Distinct roles of microRNA-1 and -499 in ventricular specification and functional maturation of human embryonic stem cell-derived cardiomyocytes. PLOS ONE 6, e27417 (2011).

153. Poon, E. N. Y. et al. Integrated transcriptomic and regulatory network analyses identify microRNA-200c as a novel repressor of human pluripotent stem cellderived cardiomyocyte differentiation and maturation. Cardiovasc. Res. 114, 894-906 (2018).

154. Lee, D. S. et al. Defined microRNAs induce aspects of maturation in mouse and human embryonic-stem-cell-derived cardiomyocytes. Cell Rep. 12, 1960-1967 (2015).

155. Bassat, E. et al. The extracellular matrix protein agrin promotes heart regeneration in mice. Nature 547, 179-184 (2017).

156. Zhou, Y. et al. Comparative gene expression analyses reveal distinct molecular signatures between differentially reprogrammed cardiomyocytes. Cell Rep. 20, 3014-3024 (2017).

157. Moroni, L. et al. Biofabrication strategies for $3 D$ in vitro models and regenerative medicine. Nat. Rev. Mater. 3, 21-37 (2018).

158. Slaughter, B. V., Khurshid, S. S., Fisher, O. Z., Khademhosseini, A. \& Peppas, N. A. Hydrogels in regenerative medicine. Adv. Mater. 21, 3307-3329 (2009)

159. Malda, J. et al. 25th anniversary article: engineering hydrogels for biofabrication Adv. Mater. 25, 5011-5028 (2013).

160. Lee, S. et al. Contractile force generation by 3D hiPSC-derived cardiac tissues is enhanced by rapid establishment of cellular interconnection in matrix with muscle-mimicking stiffness. Biomaterials 131, 111-120 (2017).

161. Kerscher, P. et al. Direct hydrogel encapsulation of pluripotent stem cells enables ontomimetic differentiation and growth of engineered human heart tissues. Biomaterials 83, 383-395 (2016).

162. Grover, G. N., Rao, N. \& Christman, K. L. Myocardial matrix-polyethylene glycol hybrid hydrogels for tissue engineering. Nanotechnology 25, 014011 (2014).

163. Bajaj, P., Schweller, R. M., Khademhosseini, A., West, J. L. \& Bashir, R. 3D biofabrication strategies for tissue engineering and regenerative medicine. Annu. Rev. Biomed. Eng. 16, 247-276 (2014)

164. Rodriguez, M. L. et al. Measuring the contractile forces of human induced pluripotent stem cell-derived cardiomyocytes with arrays of microposts. J. Biomech. Eng. 136, 051005 (2014).

165. Gilbert, T. W., Sellaro, T. L. \& Badylak, S. F. Decellularization of tissues and organs. Biomaterials 27, 3675-3683 (2006).

166. Crapo, P. M., Gilbert, T. W. \& Badylak, S. F. An overview of tissue and whole organ decellularization processes. Biomaterials 32, 3233-3243 (2011)

167. Ott, H. C. et al. Perfusion-decellularized matrix: using nature's platform to engineer a bioartificial heart. Nat. Med. 14, 213-221 (2008).

168. Oberwallner, B. et al. Preparation of cardiac extracellular matrix scaffolds by decellularization of human myocardium. J. Biomed. Mater. Res. A 102, 3263-3272 (2014)

169. Pati, F. et al. Printing three-dimensional tissue analogues with decellularized extracellular matrix bioink. Nat. Commun. 5, 1-11 (2014).

170. Perea-Gil, I. et al. A cell-enriched engineered myocardial graft limits infarct size and improves cardiac function: pre-clinical study in the porcine myocardial infarction model. JACC Basic Transl. Sci. 1, 360-372 (2016).

171. Seif-Naraghi, S. B., Salvatore, M. A., Schup-Magoffin, P. J., Hu, D. P. \& Christman K. L. Design and characterization of an injectable pericardial matrix gel: a potentially autologous scaffold for cardiac tissue engineering. Tissue Eng. Part $A$ 16, 2017-2027 (2010).

172. Johnson, T. D. et al. Human versus porcine tissue sourcing for an injectable myocardial matrix hydrogel. Biomater. Sci. 2, 735-744 (2014).

173. Bejleri, D. et al. A bioprinted cardiac patch composed of cardiac-specific extracellular matrix and progenitor cells for heart repair. Adv. Healthc. Mater. 7, 1800672 (2018)

174. Noor, N. et al. 3D printing of personalized thick and perfusable cardiac patches and hearts. Adv. Sci. 6, 1900344 (2019).

175. Zhao, Y. et al. A platform for generation of chamber-specific cardiac tissues and disease modeling. Cell 176, 913-927 (2019).
176. Engelmayr, G. C. et al. Accordion-like honeycombs for tissue engineering of cardiac anisotropy. Nat. Mater. 7, 1003-1010 (2008).

177. Tian, B. et al. Macroporous nanowire nanoelectronic scaffolds for synthetic tissues. Nat. Mater. 11, 986-994 (2012).

178. Feiner, R. et al. Engineered hybrid cardiac patches with multifunctional electronics for online monitoring and regulation of tissue function. Nat. Mater. 15, 679-685 (2016).

179. Chiu, L. L. Y., Montgomery, M., Liang, Y., Liu, H. \& Radisic, M. Perfusable branching microvessel bed for vascularization of engineered tissues. Proc. Natl Acad. Sci. USA 109, E3414-E3423 (2012).

180. Zhang, B. et al. Biodegradable scaffold with built-in vasculature for organ-on-achip engineering and direct surgical anastomosis. Nat. Mater. 15, 669-678 (2016).

181. Gouveia, P. J. et al. Flexible nanofilms coated with aligned piezoelectric microfibers preserve the contractility of cardiomyocytes. Biomaterials 139, 213-228 (2017).

182. Badrossamay, M. R., Mcllwee, H. A., Goss, J. A. \& Parker, K. K. Nanofiber assembly by rotary jet-spinning. Nano Lett. 10, 2257-2261 (2010).

183. Castilho, M. et al. Melt electrowriting allows tailored microstructural and mechanical design of scaffolds to advance functional human myocardial tissue formation. Adv. Funct. Mater. 28, 1803151 (2018).

184. Murphy, S. V. \& Atala, A. 3D bioprinting of tissues and organs. Nat. Biotechnol. 32, 773-785 (2014).

185. Hinton, T. J. et al. Three-dimensional printing of complex biological structures by freeform reversible embedding of suspended hydrogels. Sci. Adv. 1, e1500758 (2015).

186. Lee, A. et al. 3D bioprinting of collagen to rebuild components of the human heart. Science 365, 482-487 (2019).

187. Zhang, Y. S. et al. Bioprinting 3D microfibrous scaffolds for engineering endothelialized myocardium and heart-on-a-chip. Biomaterials 110, 45-59 (2016).

188. Kolesky, D. B., Homan, K. A., Skylar-Scott, M. A. \& Lewis, J. A. Three-dimensional bioprinting of thick vascularized tissues. Proc. Natl Acad. Sci. USA 113, 3179-3184 (2016).

189. Grigoryan, B. et al. Multivascular networks and functional intravascular topologies within biocompatible hydrogels. Science 364, 458-464 (2019).

190. Skylar-Scott, M. A. et al. Biomanufacturing of organ-specific tissues with high cellular density and embedded vascular channels. Sci. Adv. 5, eaaw2459 (2019).

191. Bernal, P. N. et al. Volumetric bioprinting of complex living-tissue constructs within seconds. Adv. Mater. 31, 1904209 (2019).

192. Fordyce, C. B. et al. Cardiovascular drug development: is it dead or just hibernating? J. Am. Coll. Cardiol. 65, 1567-1582 (2015).

193. Braam, S. R., Passier, R. \& Mummery, C. L. Cardiomyocytes from human pluripotent stem cells in regenerative medicine and drug discovery. Trends Pharmacol. Sci. 30, 536-545 (2009).

194. Magdy, T., Schuldt, A. J. T., Wu, J. C., Bernstein, D. \& Burridge, P. W. Human induced pluripotent stem cell (hiPSC)-derived cells to assess drug cardiotoxicity: opportunities and problems. Annu. Rev. Pharmacol. Toxicol. 58, 83-103 (2018).

195. Polonchuk, L. et al. Cardiac spheroids as promising in vitro models to study the human heart microenvironment. Sci. Rep. 7, 1-12 (2017).

196. Agarwal, A., Goss, J. A., Cho, A., McCain, M. L. \& Parker, K. K. Microfluidic heart on a chip for higher throughput pharmacological studies. Lab Chip 13, 3599-3608 (2013).

197. Marsano, A. et al. Beating heart on a chip: a novel microfluidic platform to generate functional 3D cardiac microtissues. Lab Chip 16, 599-610 (2016).

198. Feric, N. T. et al. Engineered cardiac tissues generated in the biowire II: a platform for human-based drug discovery. Toxicol. Sci. 172, 89-97 (2019).

199. Chramiec, A. et al. Integrated human organ-on-a-chip model for predictive studies of anti-tumor drug efficacy and cardiac safety. Lab Chip 20, 4357-4372 (2020).

200. Lind, J. U. et al. Cardiac microphysiological devices with flexible thin-film sensors for higher-throughput drug screening. Lab Chip 17, 3692-3703 (2017).

201. Zhao, Y. et al. A multimaterial microphysiological platform enabled by rapid casting of elastic microwires. Adv. Healthc. Mater. 8, 1801187 (2019).

202. Zhang, B. \& Radisic, M. Organ-on-A-chip devices advance to market. Lab Chip 17, 2395-2420 (2017).

203. Sun, N. et al. Patient-specific induced pluripotent stem cells as a model for familial dilated cardiomyopathy. Sci. Transl. Med. 4, 130ra47 (2012).

204. Burridge, P. W. et al. Human induced pluripotent stem cell-derived cardiomyocytes recapitulate the predilection of breast cancer patients to doxorubicininduced cardiotoxicity. Nat. Med. 22, 547-556 (2016).

205. Lan, F. et al. Abnormal calcium handling properties underlie familial hypertrophic cardiomyopathy pathology in patient-specific induced pluripotent stem cells. Cell Stem Cell 12, 101-113 (2013).

206. Lee, J. et al. Activation of PDGF pathway links LMNA mutation to dilated cardiomyopathy. Nature 572, 335-340 (2019). 
207. Te Riele, A. S. J. M. et al. Multilevel analyses of SCN5A mutations in arrhythmogenic right ventricular dysplasia/cardiomyopathy suggest non-canonical mechanisms for disease pathogenesis. Cardiovasc. Res. 113, 102-111 (2017).

208. Kodo, K. et al. iPSC-derived cardiomyocytes reveal abnormal TGF- $\beta$ signalling in left ventricular non-compaction cardiomyopathy. Nat. Cell Biol. 18, 1031-1042 (2016).

209. Itzhaki, I. et al. Modelling the long QT syndrome with induced pluripotent stem cells. Nature 471, 225-230 (2011).

210. Yazawa, M. et al. Using induced pluripotent stem cells to investigate cardiac phenotypes in Timothy syndrome. Nature 471, 230-236 (2011).

211. Matsa, E. et al. Transcriptome profiling of patient-specific human iPSCcardiomyocytes predicts individual drug safety and efficacy responses in vitro. Cell Stem Cell 19, 311-325 (2016).

212. Sharma, A. et al. High-throughput screening of tyrosine kinase inhibitor cardiotoxicity with human induced pluripotent stem cells. Sci. Transl. Med. 9, eaaf2584 (2017).

213. Smith, J. G. W. et al. Isogenic pairs of hiPSC-CMs with hypertrophic cardiomyopathy/LVNC-associated ACTC1 E99K mutation unveil differential functional deficits. Stem Cell Rep. 11, 1226-1243 (2018).

214. de la Roche, J. et al. Comparing human iPSC-cardiomyocytes versus HEK293T cells unveils disease-causing effects of Brugada mutation A735V of NaV1.5 sodium channels. Sci. Rep. 9, 1-14 (2019).

215. Wei, H., Zhang, X. H., Clift, C., Yamaguchi, N. \& Morad, M. CRISPR/Cas9 gene editing of RyR2 in human stem cell-derived cardiomyocytes provides a novel approach in investigating dysfunctional $\mathrm{Ca}^{2+}$ signaling. Cell Calcium 73, 104-111 (2018)

216. Mosqueira, D. et al. CRISPR/Cas9 editing in human pluripotent stem cellcardiomyocytes highlights arrhythmias, hypocontractility, and energy depletion as potential therapeutic targets for hypertrophic cardiomyopathy. Eur. Heart J. 39, 3879-3892 (2018).

217. Hinson, J. T. et al. Titin mutations in iPS cells define sarcomere insufficiency as a cause of dilated cardiomyopathy. Science 349, 982-986 (2015).

218. Wang, G. et al. Modeling the mitochondrial cardiomyopathy of Barth syndrome with induced pluripotent stem cell and heart-on-chip technologies. Nat. Med. 20, 616-623 (2014)

219. Goldfracht, I. et al. Generating ring-shaped engineered heart tissues from ventricular and atrial human pluripotent stem cell-derived cardiomyocytes. Nat. Commun. 11, 1-15 (2020).

220. McCain, M. L., Sheehy, S. P., Grosberg, A., Goss, J. A. \& Parker, K. K. Recapitulating maladaptive, multiscale remodeling of failing myocardium on a chip. Proc. Natl Acad. Sci. USA 110, 9770-9775 (2013).

221. Sadeghi, A. H. et al. Engineered 3D cardiac fibrotic tissue to study fibrotic remodeling. Adv. Healthc. Mater. 6, 1601434 (2017).

222. Zhang, Y. S. et al. Bioprinted thrombosis-on-a-chip. Lab Chip 16, 4097-4105 (2016).

223. Vikhorev, P. G. et al. Abnormal contractility in human heart myofibrils from patients with dilated cardiomyopathy due to mutations in TTN and contractile protein genes. Sci. Rep. 7, 1-11 (2017).

224. Prondzynski, M. et al. Disease modeling of a mutation in a-actinin 2 guides clinical therapy in hypertrophic cardiomyopathy. EMBO Mol. Med. 11, e11115 (2019).

225. Madonna, R. et al. Position Paper of the European Society of Cardiology Working Group Cellular Biology of the Heart: cell-based therapies for myocardial repair and regeneration in ischemic heart disease and heart failure. Eur. Heart J. 37, 1789-1798 (2016).

226. Sluijter, J. P. G. et al. Extracellular vesicles in diagnostics and therapy of the ischaemic heart: Position Paper from the Working Group on Cellular Biology of the Heart of the European Society of Cardiology. Cardiovasc. Res. 114, 19-34 (2018).

227. Eschenhagen, T. et al. Cardiomyocyte regeneration: a consensus statement. Circulation 136, 680-686 (2017).

228. Terrovitis, J. et al. Noninvasive quantification and optimization of acute cell retention by in vivo positron emission tomography after intramyocardial cardiac-derived stem cell delivery. J. Am. Coll. Cardiol. 54, 1619-1626 (2009).

229. Gaetani, R. et al. Cardiospheres and tissue engineering for myocardial regeneration: potential for clinical application. J. Cell. Mol. Med. 14, 1071-1077 (2010).

230. Hou, D. et al. Radiolabeled cell distribution after intramyocardial, intracoronary, and interstitial retrograde coronary venous delivery: implications for current clinical trials. Circulation 112, I-150-I-156 (2005).

231. Riegler, J. et al. Human engineered heart muscles engraft and survive long term in a rodent myocardial infarction model. Circ. Res. 117, 720-730 (2015).

232. Pecha, S. et al. Human iPS cell-derived engineered heart tissue does not affect ventricular arrhythmias in a guinea pig cryo-injury model. Sci. Rep. 9, 1-12 (2019).

233. Weinberger, F. et al. Cardiac repair in guinea pigs with human engineered heart tissue from induced pluripotent stem cells. Sci. Transl. Med. 8, 363ra148 (2016).
234. Gao, L. et al. Large cardiac muscle patches engineered from human inducedpluripotent stem cell-derived cardiac cells improve recovery from myocardial infarction in swine. Circulation 137, 1712-1730 (2018).

235. Mawad, D. et al. A conducting polymer with enhanced electronic stability applied in cardiac models. Sci. Adv. 2, e1601007 (2016).

236. Ye, L. et al. Cardiac repair in a porcine model of acute myocardial infarction with human induced pluripotent stem cell-derived cardiovascular cells. Cell Stem Cell 15, 750-761 (2014)

237. Gaetani, R. et al. Epicardial application of cardiac progenitor cells in a 3D-printed gelatin/hyaluronic acid patch preserves cardiac function after myocardial infarction. Biomaterials 61, 339-348 (2015).

238. Feyen, D. A. M. et al. Gelatin microspheres as vehicle for cardiac progenitor cells delivery to the myocardium. Adv. Healthc. Mater. 5, 1071-1079 (2016).

239. $\mathrm{Li}$, J. et al. Human pluripotent stem cell-derived cardiac tissue-like constructs for repairing the infarcted myocardium. Stem Cell Rep. 9, 1546-1559 (2017).

240. Kawamura, M. et al. Feasibility, safety, and therapeutic efficacy of human induced pluripotent stem cell-derived cardiomyocyte sheets in a porcine ischemic cardiomyopathy model. Circulation 126, S29-S37 (2012).

241. Landa, N. et al. Effect of injectable alginate implant on cardiac remodeling and function after recent and old infarcts in rat. Circulation 117, 1388-1396 (2008).

242. Seif-Naraghi, S. B. et al. Safety and efficacy of an injectable extracellular matrix hydrogel for treating myocardial infarction. Sci. Transl. Med. 5, 173 ra25 (2013).

243. Lin, X. et al. A viscoelastic adhesive epicardial patch for treating myocardial infarction. Nat. Biomed. Eng. 3, 632-643 (2019).

244. Almeida, S. O., Skelton, R. J., Adigopula, S. \& Ardehali, R. Arrhythmia in stem cell transplantation. Card. Electrophysiol. Clin. 7, 357-370 (2015).

245. Nguyen, P. K., Neofytou, E., Rhee, J. W. \& Wu, J. C. Potential strategies to address the major clinical barriers facing stem cell regenerative therapy for cardiovascular disease: a review. JAMA Cardiol. 1, 953-962 (2016)

246. Madonna, R. Human-induced pluripotent stem cells: in quest of clinical applications. Mol. Biotechnol. 52, 193-203 (2012).

247. Breitbach, M. et al. Potential risks of bone marrow cell transplantation into infarcted hearts. Blood 110, 1362-1369 (2007).

248. Blin, G. et al. A purified population of multipotent cardiovascular progenitors derived from primate pluripotent stem cells engrafts in postmyocardial infarcted nonhuman primates. J. Clin. Invest. 120, 1125-1139 (2010).

249. Yoon, Y. S., Park, J. S., Tkebuchava, T., Luedeman, C. \& Losordo, D. W. Unexpected severe calcification after transplantation of bone marrow cells in acute myocardial infarction. Circulation 109, 3154-3157 (2004).

250. Chung, L., Maestas, D. R., Housseau, F. \& Elisseeff, J. H. Key players in the immune response to biomaterial scaffolds for regenerative medicine. Adv. Drug Deliv. Rev. 114, 184-192 (2017).

251. Menasché, P. et al. Transplantation of human embryonic stem cell-derived cardiovascular progenitors for severe ischemic left ventricular dysfunction. J. Am. Coll. Cardiol. 71, 429-438 (2018).

252. Mewhort, H. E. M. et al. Epicardial infarct repair with bioinductive extracellular matrix promotes vasculogenesis and myocardial recovery. J. Heart Lung Transplant. 35, 661-670 (2016).

253. Yanagawa, B., Rao, V., Yau, T. M. \& Cusimano, R. J. Potential myocardial regeneration with cormatrix ECM: a case report. J. Thorac. Cardiovasc. Surg. 147, e41-e43 (2014).

254. Mewhort, H. E. M., Turnbull, J. D., Meijndert, H. C., Ngu, J. M. C. \& Fedak, P. W. M. Epicardial infarct repair with basic fibroblast growth factor-enhanced CorMatrixECM biomaterial attenuates postischemic cardiac remodeling. J. Thorac. Cardiovasc. Surg. 147, 1650-1659 (2014).

255. Traverse, J. H. et al. First-in-man study of a cardiac extracellular matrix hydrogel in early and late myocardial infarction patients. JACC Basic Transl. Sci. 4, 659-669 (2019).

256. Frey, N. et al. Intracoronary delivery of injectable bioabsorbable scaffold (IK$5001)$ to treat left ventricular remodeling after ST-elevation myocardial infarction: a first-in-man study. Circ. Cardiovasc. Interv. 7, 806-812 (2014).

257. Rao, S. V. et al. Bioabsorbable intracoronary matrix for prevention of ventricular remodeling after myocardial infarction. J. Am. Coll. Cardiol. 68, 715-723 (2016).

258. Rao, S. V. et al. A randomized, double-blind, placebo-controlled trial to evaluate the safety and effectiveness of intracoronary application of a novel bioabsorbable cardiac matrix for the prevention of ventricular remodeling after large ST-segment elevation myocardial infarction: Rationale and design of the PRESERVATION I trial. Am. Heart J. 170, 929-937 (2015).

259. Anker, S. D. et al. A prospective comparison of alginate-hydrogel with standard medical therapy to determine impact on functional capacity and clinical outcomes in patients with advanced heart failure (AUGMENT-HF trial). Eur. Heart J. 36, 2297-2309 (2015).

260. Mann, D. L. et al. One-year follow-up results from AUGMENT-HF: a multicentre randomized controlled clinical trial of the efficacy of left ventricular augmentation with Algisyl in the treatment of heart failure. Eur. J. Heart Fail. 18, 314-325 (2016). 
261. Lee, R. J. et al. The feasibility and safety of Algisyl-LVR ${ }^{\mathrm{TM}}$ as a method of left ventricular augmentation in patients with dilated cardiomyopathy: initial first in man clinical results. Int. J. Cardiol. 199, 18-24 (2015).

262. Puymirat, E. et al. Can mesenchymal stem cells induce tolerance to cotransplanted human embryonic stem cells? Mol. Ther. 17, 176-182 (2009).

\section{ACKNOWLEDGEMENTS}

We acknowledge funding received from EU-funded project BRAV3 (H2020, ID:874827); Fundação para a Ciência e Tecnologia (FCT)-funded projects NETDIAMOND (SAICTPAC) 0047/2015), MetaCardio (PTDC/BTMSAL/32566/2017) and Neocoronary (PTDC/MECCAR/29590/2017); iNOVA4Health, refs. UIDB/04462/2020 and UIDP/04462/2020, a program financially supported by FCT/Ministério da Educação e Ciência, through national funds. A.F.L. was supported by FCT Grant PD/BD/139078/2018.

\section{AUTHOR CONTRIBUTIONS}

M.F.T. and A.F.L. wrote the manuscript. P.M.A. and M.S. reviewed and edited the manuscript and were responsible for funding acquisition.

\section{COMPETING INTERESTS}

The authors declare no competing interests.

\section{ADDITIONAL INFORMATION}

Reprints and permission information is available at http://www.nature.com/ reprints

Publisher's note Springer Nature remains neutral with regard to jurisdictional claims in published maps and institutional affiliations.

(i) Open Access This article is licensed under a Creative Commons Attribution 4.0 International License, which permits use, sharing, adaptation, distribution and reproduction in any medium or format, as long as you give appropriate credit to the original author(s) and the source, provide a link to the Creative Commons license, and indicate if changes were made. The images or other third party material in this article are included in the article's Creative Commons license, unless indicated otherwise in a credit line to the material. If material is not included in the article's Creative Commons license and your intended use is not permitted by statutory regulation or exceeds the permitted use, you will need to obtain permission directly from the copyright holder. To view a copy of this license, visit http://creativecommons. org/licenses/by/4.0/.

(C) The Author(s) 2021 